\title{
Characterization of an RF-driven argon plasma at atmospheric pressure using broadband absorption and optical emission spectroscopy
}

\author{
G. Nayak, ${ }^{1,}$ a) M. Simeni Simeni, ${ }^{1}$ J. Rosato, ${ }^{2}$ N. Sadeghi, ${ }^{3, \text { b) }}$ and P. J. Bruggeman ${ }^{1, c)}$ \\ ${ }^{1)}$ Department of Mechanical Engineering, University of Minnesota, Minneapolis, Minnesota 55455, \\ USA \\ 2)Aix-Marseille Université, CNRS, PIIM UMR7345, 13397 Marseille, France \\ ${ }^{3)}$ LIPhy (URA5588) \& LTM (URA5129), Université Grenoble-Alpes \& CNRS, Grenoble, \\ France
}

(Dated: 30 November 2020)

\begin{abstract}
Atmospheric pressure plasmas in argon are of particular interest due to the production of highly excited and reactive species enabling numerous plasma-aided applications. In this contribution, we report on absolute optical emission and absorption spectroscopy of a radio frequency (RF) driven capacitively coupled argon glow discharge operated in a parallel-plate configuration. This enabled the study of all key parameters including electron density and temperature, gas temperature, and absolute densities of atoms in highly electronically excited states. The space and time-averaged electron density and temperature were determined from the measurement of the absolute intensity of the electron-atom bremsstrahlung in the visible range. Considering the non-Maxwellian electron energy distribution function, an electron temperature $\left(T_{\mathrm{e}}\right)$ of $2.1 \mathrm{eV}$ and an electron density $\left(n_{\mathrm{e}}\right)$ of $1.1 \times 10^{19} \mathrm{~m}^{-3}$ were obtained. The time-averaged and spatially resolved absolute densities of atoms in the metastable $\left(1 s_{5}\right.$ and $\left.1 s_{3}\right)$ and resonant $\left(1 s_{4}\right.$ and $\left.1 s_{2}\right)$ states of argon in pure Ar and $\mathrm{Ar} / \mathrm{He}$ mixture were obtained by broadband absorption spectroscopy. The $1 s_{5}$ metastable atoms had the largest density near the sheath region with a maximum value of $8 \times 10^{17} \mathrm{~m}^{-3}$, while all other $1 s$ states had densities of at most $2 \times 10^{17} \mathrm{~m}^{-3}$. The dominant production and loss mechanisms of these atoms were discussed, in particular, the role of radiation trapping. We conclude with a comparison of the plasma properties of the argon RF glow discharges with the more common He equivalent and highlight their differences.
\end{abstract}

\section{INTRODUCTION}

Plasmas operating at atmospheric pressure in argon have witnessed a tremendous growth in last few decades. The nonequilibrium feature of such discharges allows production of highly excited and reactive species initiated by energetic electrons, which have enabled a plethora of applications, including gas discharge laser systems such as optically pumped rare gas lasers (OPRGLs) operated in Ar/He mixtures, ${ }^{1-5}$ gas discharge lamps, plasma medicine ${ }^{6-8}$ surface modification, ${ }^{9-11}$ and surface decontamination. ${ }^{12-14}$ In highly repetitive discharges, such as radio frequency (RF) driven plasmas, the long-lived charged and energetic species from the previous discharge cycle enable to sustain stable discharges at lower voltage requirements. The argon metastable species are one of those strong contenders for driving the kinetics in these low temperature plasmas. ${ }^{15}$ Due to their relatively long lifetime, atoms in the metastable states are known to be excellent reservoirs of energy. The excitation energy required for metastable production $(11.6 \mathrm{eV})$ is lower than the ionization energy of argon $(15.8 \mathrm{eV})$. Thus, these metastable atoms can be produced efficiently and can further lead to ionization at lower electron energy via stepwise ionization.

Since argon is used as a feed gas for many plasma-aided applications, it becomes imperative to study its metastable states in terms of the absolute densities and the spatial distribution within the active plasma region. Numerous mod-

\footnotetext{
a)Electronic mail: nayak025@umn.edu

b)Electronic mail: nader.sadeghi@univ-grenoble-alpes.fr

c)Electronic mail: pbruggem@umn.edu
}

eling studies have been carried out to understand the kinetics of the argon metastable atoms in $\mathrm{Ar}$ and $\mathrm{Ar} / \mathrm{He}$ plasmas over a wide range of pressures (few mTorrs to atmospheric pressure). ${ }^{2,16-20}$ However, only a handful of papers report measurements of these vital species at atmospheric pressure. Most of the argon metastable species measurements are performed using tunable diode laser absorption spectroscopy ${ }^{4,5,21-25}$ or time-resolved laser induced fluorescence measurements. ${ }^{1}$ In addition, the resonant states of argon could also be important energy reservoirs, but they have only been studied in low pressure argon plasmas. ${ }^{26-28}$ At atmospheric pressure, the effective lifetimes of the metastable states of Ar, $1 s_{5}$ and $1 s_{3}$ (Paschen's notation, details provided further in Table II), are governed by three-body collisions with two ground state $\mathrm{Ar}$ atoms, producing $\mathrm{Ar}_{2}^{*}$ excimers, and by collisional transfer to the resonant states, $1 s_{2}$ and $1 s_{4}$ via both electron-impact and heavy particle collisions. ${ }^{28,29}$ A more detailed measurement/analysis of the population of these resonant states will facilitate an understanding of the dominant energy transfer mechanism(s) and its potential impact on plasma kinetics.

In this work, the time-averaged absolute densities of Ar atoms in both metastable and resonant states of Ar in pure $\mathrm{Ar}$ and $\mathrm{Ar} / \mathrm{He}$ gas mixtures in an RF-driven capacitively coupled glow discharge at atmospheric pressure are measured using broadband absorption spectroscopy. The electron temperature and density are determined from continuum radiation emission spectroscopy and compared with the electron density obtained from a previously reported electrical impedance measurement approach. ${ }^{30}$ The experimental setup, optical diagnostics and a detailed procedure to determine the electron temperature and density as well as the absolute densities from 
the measured absorption data are described before we present the results. The estimated electron properties and the spatially distributed densities of atoms in the metastable and resonant states of Ar across the plasma gap allow to discuss the production and destruction mechanisms of the excited atoms of Ar in detail.

\section{METHODS}

\section{A. Experimental setup}

A schematic of the experimental setup is shown in Fig. 1(a). The plasma reactor used in this study generates an RF-driven capacitively coupled glow discharge in a water-cooled parallel plate electrode type configuration. A detailed description of the reactor is provided $\mathrm{in}^{31,32}$. The reactor is designed to facilitate access for optical diagnostics along the length of the plasma (19.1 mm) and within the inter-electrode spacing of 2 $\mathrm{mm}$. The discharge was generated at atmospheric pressure in argon (ultra-pure carrier grade $99.999 \%$ ) as well as in helium (ultra-pure carrier grade $99.9995 \%$ ) with $17 \%$ admixture of argon to elucidate on the effect of absolute densities of atoms in the metastable and resonant states of Ar with $\mathrm{He}$ as the perturber atom. The total flow rate of the gas or gas mixture used was maintained using electronic mass flow controllers (MFCs) at 1 standard liter per minute (slm) for Ar and $\mathrm{He}+$ $17 \%$ Ar plasmas. The time-averaged plasma images for different gas conditions were obtained using a digital camera with a $1 / 10$ s exposure time.

The RF power $(13.56 \mathrm{MHz})$ was applied continuously and only time-averaged densities of metastable and resonant atoms are reported. The discharge power was determined by acquiring and recording voltage and current data on a digital oscilloscope (Tektronix DPO2024B, $200 \mathrm{MHz}, 1 \mathrm{Gs}^{-1}$ ) using a voltage probe (Tektronix P5100A) and a Rogowski coil (Pearson Electronics, Model 2878), respectively as shown in Fig. 1(a). The discharge powers used for different gas mixture are reported in Table $\mathrm{I}$.

\section{B. Spectroscopy of emission continuum}

The continuum radiation, visible in pure Ar discharge (Fig. 1(b)), mostly originates from collision-induced processes, for example, free-free bremsstrahlung and free-bound recombination between electrons and ions in low pressure plasmas. ${ }^{33}$ However, in weakly ionized plasmas with a low degree of ionization $\left(n_{\mathrm{e}} / n_{\mathrm{a}}<10^{-4}, n_{\mathrm{a}}\right.$ being the density of the neutral atom), such as the atmospheric pressure glow discharges studied in this work, the continuum radiation emitted through interactions between free electrons and neutral atoms becomes dominant over the electron-ion interactions typical for high density plasmas, where Coulomb collisions dominate. ${ }^{34}$ This "continuum" radiation is further composed of two major contributing mechanisms: (a) the electronneutral atom $(e-a)$ or neutral bremsstrahlung, caused by the deceleration of a free electron during its interaction with a
TABLE I. Discharge power for different plasma conditions.

\begin{tabular}{lcr}
\hline \hline Gas & $\begin{array}{c}\text { Flow rate } \\
(\text { slm })\end{array}$ & $\begin{array}{r}\text { Power } \\
(\mathrm{W})\end{array}$ \\
\hline $\mathrm{Ar}$ & 1 & $13.5 \pm 0.2$ \\
$\mathrm{He}$ & 1 & $14.0 \pm 0.5$ \\
$\mathrm{He}+17 \% \mathrm{Ar}$ & 1 & $12.4 \pm 1.4$ \\
\hline \hline
\end{tabular}

neutral atom, and (b) the polarization bremsstrahlung, which originates from the time-dependent induced dipole moment in neutral atom by the Coulomb field of the incident electron. However, it is shown that for electron-argon collisions with $T_{\mathrm{e}}>0.9 \mathrm{eV}$, the neutral bremsstrahlung spectral intensities are much higher than that of polarization bremsstrahlung. ${ }^{35}$ This was further confirmed by numerical studies in ${ }^{36}$, which showed that the induced dipole radiation contributes to $<8 \%$ of the total radiation for a $T_{\mathrm{e}}=0.5 \mathrm{eV}$, and was insignificant in most cases. Thus, in this study, the continuum radiation will be analyzed and discussed based on neutral bremsstrahlung only.

The emission from the continuum radiation is usually studied by determining the emissivity as function of wavelength. The emissivity of the $e$ - $a$ bremsstrahlung has been reported in detail in ${ }^{37}$ and can be expressed as

$$
\varepsilon_{\mathrm{ea}}=4 \pi \times \sqrt{\frac{2}{m_{\mathrm{e}}}} \frac{n_{\mathrm{e}} n_{\mathrm{a}}}{\lambda^{2}} \frac{h c}{4 \pi} \int_{h v}^{\infty} \sigma_{\mathrm{ea}}^{\mathrm{B}}(\lambda, E) \sqrt{E} f(E) \mathrm{d} E,
$$

where $m_{\mathrm{e}}, \lambda, h, c, h v$ and $E$ are the mass of an electron, the wavelength of the emitted photon, the Planck constant, the speed of light in vacuum, the energy of the emitted photon and the energy of the electron, respectively. In this work, we use SI units and $\varepsilon_{\text {ea }}$ has the units $\left[\mathrm{W} \mathrm{m} \mathrm{m}^{-3} \mathrm{~nm}^{-1}\right]$. The electron energy distribution function (EEDF), $f(E)$ is derived from Bolsig $+{ }^{38}$ and both Maxwellian as well as non-Maxwellian distributions are considered to compare their effect on the estimation of $T_{\mathrm{e}}$ and $n_{\mathrm{e}}$. The neutral bremsstrahlung cross-section $\sigma_{\text {ea }}^{\mathrm{B}}(\lambda, E)$ according to ${ }^{39}$ is given by

$$
\sigma_{\mathrm{ea}}^{\mathrm{B}}(\lambda, E)=\frac{8 \alpha}{3 \pi} \frac{E}{m_{\mathrm{e}} c^{2}}\left(1-\frac{h c}{2 \lambda E}\right) \sqrt{1-\frac{h c}{\lambda E}} \sigma_{\mathrm{ea}}^{\mathrm{mom}}(E),
$$

where $\sigma_{\text {ea }}^{\text {mom }}(E)$ is the cross-section for momentum transfer between the electron and the neutral argon atom adopted from the Phelps database in LXCat. ${ }^{38,40}$ The fine structure constant $\alpha$ is given by

$$
\alpha=\frac{e^{2}}{2 \varepsilon_{0} h c} .
$$

Substituting Eq. 2 in Eq. 1 yields

$$
\begin{aligned}
\varepsilon_{\mathrm{ea}}= & C_{\mathrm{ea}} \frac{n_{\mathrm{e}} n_{\mathrm{a}}}{\lambda^{2}} \int_{h v}^{\infty} \sigma_{\mathrm{ea}}^{\mathrm{mom}}(E) E^{3 / 2} \\
& \times\left(1-\frac{h c}{2 \lambda E}\right) \sqrt{1-\frac{h c}{\lambda E}} f(E) \mathrm{d} E,
\end{aligned}
$$




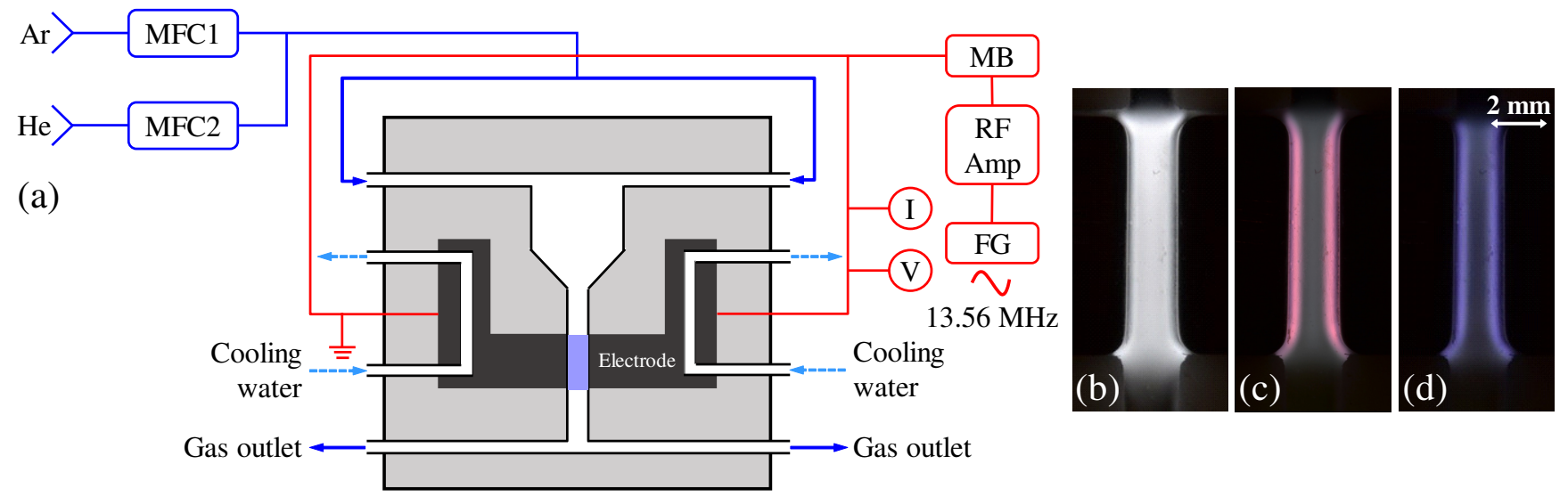

FIG. 1. (a) Experimental schematic of the plasma reactor, and digital color images of the plasma operated in (b) pure Ar, (c) pure He, and (d) $\mathrm{He}+17 \%$ Ar. (FG: function generator, RF Amp: RF amplifier, MB: matching box, MFC: mass flow controller)

where $C_{\mathrm{ea}}=\left(2 \sqrt{2} / 3 \pi^{2}\right)\left(\alpha h / m_{\mathrm{e}}^{3 / 2} c\right)=1.77\left[\mathrm{~W} \mathrm{~m} \mathrm{~m}^{2} \mathrm{~J}^{-3 / 2}\right.$ $\left.\mathrm{sr}^{-1}\right]$. The EEDF, $f(E)$ has units $\left[\mathrm{eV}^{-3 / 2}\right]$. Integrating $E^{-1 / 2} f(E)$ over the entire energy range yields 1 . Eq. 4 shows that $T_{\mathrm{e}}$ and $n_{\mathrm{e}}$ determine the spectral shape and the intensity of the neutral bremsstrahlung. Thus, by comparing the measured absolute continuum radiation with the theoretical spectrum, $T_{\mathrm{e}}$ and $n_{\mathrm{e}}$ can be determined.

\section{Determination of electron density and temperature from continuum radiation spectra}

The continuum radiation requires the measurement of the absolute spectral irradiance (in [ $\left.\mathrm{W} \mathrm{m}^{-2} \mathrm{~nm}^{-1}\right]$ ). In this work, we only determined the time and spatially averaged absolute plasma emission through an absolute calibration of the spectrometer and detection system with a tungsten halogen lamp. The optical configuration for recording the global emission from the plasma is schematically shown in Fig. 2. The plasma reactor was placed at a distance of $0.5 \mathrm{~m}$ from a diaphragm with a diameter of $4.2 \mathrm{~mm}$. The plasma emission through the diaphragm was focused into an optical fiber by a UV-visible plano-convex lens $(f=7.5 \mathrm{~cm})$, which was then analyzed by a broadband spectrometer (Avantes 2048) with a spectral resolution of $0.6 \mathrm{~nm}$. To achieve calibration, the calibrated tungsten halogen lamp (Oriel, Spectral Irradiance (250-2400 nm) 200 Watt QTH Lamp, Model No 63355) with known spectral irradiance at a distance of $500 \mathrm{~mm}$ was recorded by replacing the plasma source with the lamp and keeping all other optical components fixed and identical. At a distance of $500 \mathrm{~mm}$, both the plasma and the light source can be considered as a point source and this simplifies the further analysis significantly. The absolute spectral irradiance, $I_{\mathrm{P}}(\lambda)$ of the plasma is then given as follows

$$
I_{\mathrm{P}}(\lambda)=\frac{P(\lambda)}{L(\lambda)} I_{\mathrm{L}}(\lambda)
$$

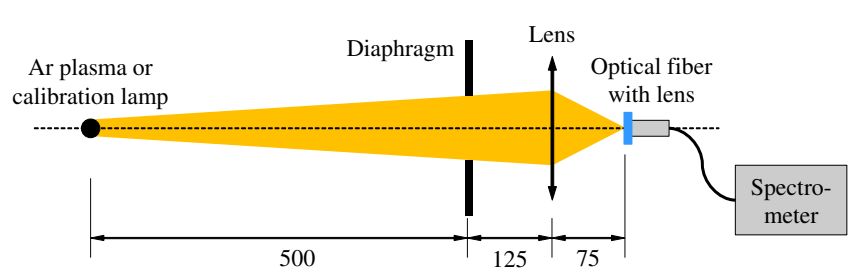

FIG. 2. Setup used for optical emission spectroscopy. The provided dimensions have $\mathrm{mm}$ units.

where $P(\lambda)$ and $L(\lambda)$ are the relative emission intensities from the plasma and the lamp, respectively, measured by the same optical detection system and $I_{\mathrm{L}}(\lambda)$ is the spectral irradiance of the calibrated tungsten lamp at the same distance of 0.5 $\mathrm{m}$. Now, the volume-averaged spectral emission coefficient or emissivity (in [ $\left.\mathrm{W} \mathrm{m}^{-3} \mathrm{~nm}^{-1}\right]$ ) of the Ar plasma can be obtained from the calibrated spectral irradiance, $I_{\mathrm{P}}(\lambda)$ by $^{41,42}$

$$
\langle j(\lambda)\rangle_{\mathrm{V}}=I_{\mathrm{P}}(\lambda) \frac{d^{2}}{V_{\mathrm{P}}}
$$

where $d$ is the distance between the light source and the diaphragm $(0.5 \mathrm{~m})$ and $V_{\mathrm{P}}$ is the plasma volume. The calculated emissivity $\varepsilon_{\text {ea }}$ from Eq. 4 is fitted to the measured spectral irradiance $\langle j(\lambda)\rangle_{\mathrm{V}}$ to obtain a spatially averaged $T_{\mathrm{e}}$ and $n_{\mathrm{e}}$. This fitting is performed in the range of $350 \mathrm{~nm} \leq \lambda \leq 900 \mathrm{~nm}$ since the spectral sensitivity of the calibrated halogen lamp drops significantly below $350 \mathrm{~nm}$ and the sensitivity of the spectrometer reduces significantly above $900 \mathrm{~nm}$.

\section{Electrical model}

An independent determination of $n_{\mathrm{e}}$ is obtained by the analysis of an equivalent electrical circuit for a homogeneous RF plasma as reported $\mathrm{in}^{30,43}$. This method is based on a simplified homogeneous RF discharge model without considering 


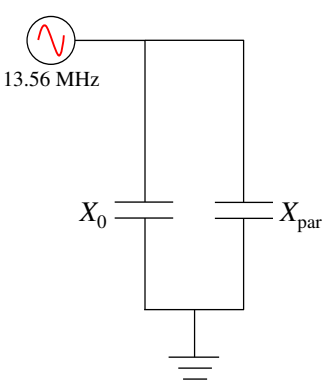

(a)

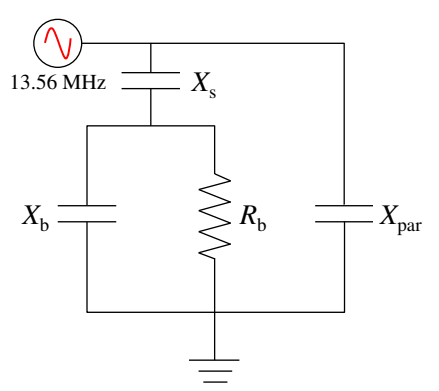

(b)
FIG. 3. Equivalent electrical circuit used for the calculations of the sheath width and the electron density with (a) plasma off, and (b) plasma on.

sheath effects (stochastic and ion heating), ${ }^{44,45}$ while isolating the influence of the bulk plasma and the sheaths. The assumptions used in this model are only valid for discharges operating in the $\alpha$-mode, which is valid for the Ar plasma investigated in this study (see also further). This electrical model allows the determination of the electrical characteristics of the bulk plasma and the sheath, such as the plasma impedance, resistance and reactance. Fig. 3 shows the equivalent electrical circuit for the RF plasma under operation. For a detailed analysis of the equivalent electrical circuit, the reader is referred to $^{43}$. Briefly, the components of the equivalent electrical circuit, such as the sheath reactance $\left(X_{\mathrm{S}}\right)$, the bulk plasma reactance $\left(X_{\mathrm{b}}\right)$ and the bulk plasma resistance $\left(R_{\mathrm{b}}\right)$ can be obtained by solving the following equations ${ }^{43}$

$$
\begin{gathered}
X_{\mathrm{s}}=X_{\mathrm{p}}+\frac{R_{\mathrm{p}}^{2}}{X_{\mathrm{p}}-X_{0}}, \\
X_{\mathrm{b}}=X_{0}-X_{\mathrm{s}}, \text { and } \\
R_{\mathrm{b}}=R_{\mathrm{p}}\left[1+\frac{R_{\mathrm{p}}^{2}}{\left(X_{\mathrm{p}}-X_{0}\right)^{2}}\right],
\end{gathered}
$$

where $X_{0}=\left(\omega C_{0}\right)^{-1}$ is the reactance of the discharge gap without plasma with capacitance $C_{0}$, and $R_{\mathrm{p}}\left(=\frac{V}{I} \cos (\Delta \phi)\right)$ and $X_{\mathrm{p}}\left(=\frac{V}{I} \sin (\Delta \phi)\right)$ are the real and imaginary parts of the plasma impedance, respectively. When the plasma is not ignited yet, the impedance should correspond to a purely capacitive reactance (Fig. 3a), with $C_{0}=0.8 \mathrm{pF}$, however, the measured plasma-off impedance corresponds to a value of $C=\left(\omega X_{\mathrm{p}}\right)^{-1}=14.6 \mathrm{pF}$. This huge deviation is caused by the additional stray or parasitic capacitances in the system, and are clubbed together as $X_{\text {par }}$ by adding an additional parasitic capacitance, $C_{\text {par }}$ parallel to the RF glow discharge in Fig. $3 \mathrm{~b}$, where $C_{\mathrm{par}}=C-C_{0}=13.8 \pm 0.1 \mathrm{pF}$. Considering $C_{\text {par }}$ in the equivalent circuit, the current through the plasma

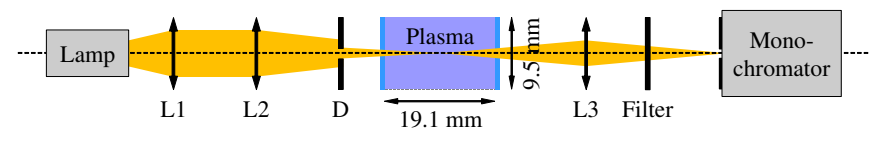

FIG. 4. Setup used for broadband absorption spectroscopy.

circuit was recalculated from the measured current. The timeaveraged electron density can then be estimated from $R_{\mathrm{b}}$ using impedance measurement and a homogeneous plasma model ${ }^{45}$

$$
n_{\mathrm{e}}=\frac{l-2 s_{0}}{A R_{\mathrm{b}}} \frac{v_{\mathrm{m}} m_{\mathrm{e}}}{e^{2}},
$$

where $l$ is the inter-electrode spacing ( $2 \mathrm{~mm}$ in this case), $s_{0}$ the time-averaged sheath width and $A$ the cross-sectional area of the electrode. The rates of electron-neutral momentum transfer $\left(v_{\mathrm{m}}\right)$ for argon and helium at atmospheric pressure are $4.03 \times 10^{12} \mathrm{~s}^{-1}$ and $1.52 \times 10^{12} \mathrm{~s}^{-1}$, respectively. ${ }^{46}$ Using the measured sheath capacitance $X_{\mathrm{s}}$, the sheath width can be estimated as follows: ${ }^{30,44}$

$$
s_{0}=\omega \varepsilon_{0} A X_{\mathrm{s}} / 2,
$$

where $\omega=2 \pi f$ is the plasma excitation frequency. The uncertainties in the time-averaged sheath width and the electron density are estimated by propagating the measurement uncertainties in $C_{\mathrm{par}}$ and $\Delta \phi_{\mathrm{p}}$ through the equations of the equivalent electrical model.

\section{E. Broadband absorption spectroscopy}

The absolute densities of Ar atoms in metastable and resonant states across the plasma gap were determined by employing broadband absorption spectroscopy. The optical configuration for the spectrally resolved measurements is similar as used in $^{31}$ and is shown in Fig. 4.

A laser-stabilized broadband lamp (Energetiq EQ-99 LDLS) was used as the light source. The light produced by the lamp was focused into the plasma reactor via lenses L1 $(f=20 \mathrm{~cm})$ and L2 $(f=10 \mathrm{~cm})$ through a diaphragm $\mathrm{D}$ with a diameter of $1 \mathrm{~mm}$. After passing the reactor, the light was immediately focused onto the entrance slit of a $1 \mathrm{~m}$ high-resolution Czerny-Turner scanning monochromator (ARC AM-510), equipped with an $1800 \mathrm{~g} \mathrm{~mm}^{-1}$ grating blazed at $500 \mathrm{~nm}$, via a plano-convex lens L3 $(f=5 \mathrm{~cm})$. The width of the entrance slit was fixed at $10 \mu \mathrm{m}$ and was located $5 \mathrm{~cm}$ from the lens L3. A neutral density filter was used in all the experimental conditions to avoid the saturation of the detector by the lamp. The spectrometer was backed by a $26 \mu \mathrm{m}$ pitch charged-coupled device (CCD) camera (Andor iDUS 420). The spectral resolution of the optical system (monochromator + CCD), as deduced from the width of the atomic line of mercury in a low pressure $\mathrm{Hg}$-Ar lamp, is 29 pm FWHM at $365 \mathrm{~nm}$ monitored in the $1^{\text {st }}$ order of diffraction. 
To obtain spatially resolved measurements, the plasma reactor was moved in steps of $50 \mu \mathrm{m}$ along the plasma gap, on a translational stage. The spatial resolution was $40 \mu \mathrm{m}$. For the determination of the spatial resolution within the plasma gap, the readers are referred to the procedure described $\mathrm{in}^{31}$.

\section{F. Gas temperature}

Due to the presence of a small concentration of water impurities in $\mathrm{Ar}$ and $\mathrm{He}+17 \%$ Ar plasmas, we were able to record high resolution rotational spectra of $\mathrm{OH}(A-X)(0-0)$. In this case, a bandpass filter (240-420 nm), instead of a neutral density filter, was used. The experimentally measured emission spectra of $\mathrm{OH}(A-X)$ in different gas conditions were fitted with synthetic spectra obtained by following the modeling procedure described in ${ }^{47}$ to determine the gas temperature. The reported uncertainties were derived from the $95 \%$ confidence interval using the least-square fitting procedure. An example of a recorded $\mathrm{OH}(A-X)$ emission spectrum along with the simulated best fit is shown in Fig. 5 for the $\mathrm{He}+17 \%$ Ar plasma. The gas temperatures obtained from the rotational temperature of $\mathrm{OH}(A-X)$ are $365 \mathrm{~K}$ and $335 \mathrm{~K}$ for pure $\mathrm{Ar}$ and $\mathrm{He}+17 \%$ Ar plasmas, respectively.

\section{G. Absolute densities of atoms in resonant and metastable states}

The different excited states and corresponding transitions investigated in this study are compiled in Table II. Density of Ar metastable atoms in $1 s_{3}$ and $1 s_{5}$ states, according to Paschen notation (or $4 s^{\prime}[1 / 2]_{0}$ and $4 s[3 / 2]_{2}$ in Racah notation), are measured from absorption on 763.51 and 794.82 $\mathrm{nm}$ transitions, respectively. Density of atoms in resonance states $1 s_{2}$ and $1 s_{4}$ (or $4 s^{\prime}[1 / 2]_{1}$ and $4 s[3 / 2]_{1}$ ), are measured by means of 750.39 and $751.47 \mathrm{~nm}$ lines, respectively. $1 s_{2}$ and $1 s_{4}$ states being optically connected to the ground state of $\mathrm{Ar}$, these later lines are strongly broadened by resonance interaction (resonance broadening).

The detailed procedure for obtaining the absorption spectrum using broadband absorption spectroscopy is reported $i^{31,48-50}$. In brief, the experimental fractional absorption $A(\lambda)$ can be calculated as follows: ${ }^{49}$

$$
A(\lambda)=1-\frac{L_{\mathrm{P}}(\lambda)-P(\lambda)}{L_{0}(\lambda)-B(\lambda)},
$$

where $P(\lambda)$ and $L_{0}(\lambda)$ are the emission spectra of the plasma and the lamp, respectively; $L_{\mathrm{P}}(\lambda)$ is the emission spectrum of the plasma with the transmitted light of the lamp; and $B(\lambda)$ is the background spectrum with both plasma and lamp off. As an example, these emission spectra were recorded for $1 s_{2}, 1 s_{3}$, $1 s_{4}$ and $1 s_{5}$ states in $\mathrm{He}+17 \%$ Ar at the sheath edge, $250 \mu \mathrm{m}$ from the powered electrode, and the corresponding fractional absorption spectra obtained using Eq. 12 are shown in Fig. 6. The absorption spectra for these states contained oscillations as reported $\mathrm{in}^{31}$, the amplitude of which were significantly

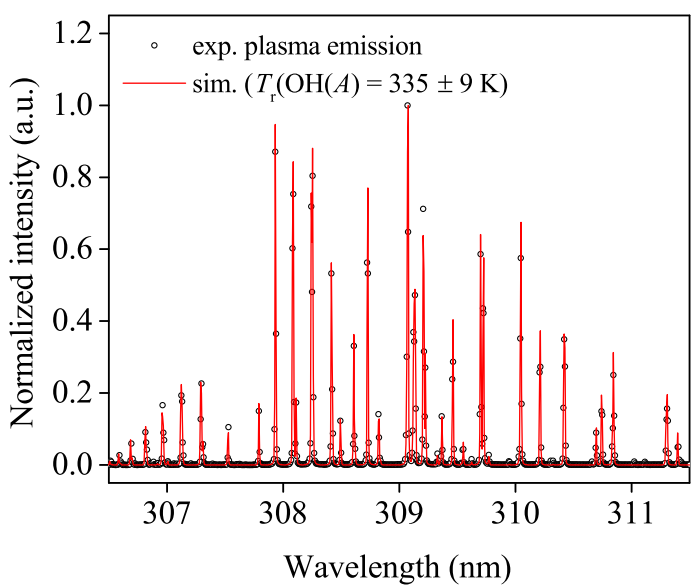

FIG. 5. Gas temperature determination from an experimentally recorded emission spectrum of $\mathrm{OH}(A-X)$ and the simulated best fit for $\mathrm{He}+17 \%$ Ar plasma operating at $12.4 \mathrm{~W}$ and measured at a distance of $600 \mu \mathrm{m}$ from the powered electrode surface.

attenuated by using FFT band block filters at frequency ranges of $0.18-0.2$ per pixel and $0.086-0.095$ per pixel. This induced an average difference of $\sim 10 \%$ in the magnitude of the peak fractional absorption without affecting the total wavelength integrated area under the absorption line profile significantly.

The absolute path-averaged density, $n$, of the absorbing species can be determined by calculating the wavelength integrated area $W$ under the fractional absorption curve for each of the above cited argon lines and using the Beer-Lambert law as follows: ${ }^{49}$

$$
W=\int A(\lambda) \mathrm{d} \lambda=\int(1-\exp (-\sigma(\lambda, T) \cdot n \cdot l)) \mathrm{d} \lambda,
$$

where $T$ is the neutral gas temperature, $\sigma$ the wavelength and temperature dependent absorption cross-section, and $l$ the beam path length through the plasma $(19.1 \mathrm{~mm})$. As previously reported in ${ }^{49}, W$ is independent of the instrumental spectral resolution, and thus, can be experimentally determined by fitting the absorption profile for each line with a Voigt function as shown in Fig. 6. In Eq. 13, the absorption cross-section, $\sigma$ $\left(\mathrm{m}^{2}\right)$, is given by

$$
\sigma(\lambda, T)=\lambda_{0}^{2} \frac{e^{2}}{4 \varepsilon_{0} m_{\mathrm{e}} c^{2}} f_{\mathrm{lu}} \varphi(\lambda)
$$

where $\lambda_{0}, e, \varepsilon_{0}, m_{\mathrm{e}}, c, f_{\mathrm{lu}}$ and $\varphi(\lambda)$ are the peak wavelength of the observed transition $(\mathrm{m})$, the elementary charge $(\mathrm{C})$, the permittivity of the free space $\left(\mathrm{F} \mathrm{m}^{-1}\right)$, the electron mass $(\mathrm{kg})$, the speed of light in vacuum $\left(\mathrm{m} \mathrm{s}^{-1}\right)$, the dimensionless oscillator strength of the line and the area-normalized spectral shape of the absorption line profile, respectively. ${ }^{49}$ In high pressure plasmas with a low ionization degree as in the glow discharges studied, collisional broadening due to neutral collisions is the dominant effect, while natural broadening is negligible. Assuming a Maxwellian velocity distribution, $\varphi(\lambda)$ 
TABLE II. Different transitions of Ar and their characteristics. ${ }^{51}$

\begin{tabular}{llllllll}
\hline \hline $\begin{array}{l}\text { Observed } \\
\text { wavelength } \\
(\mathrm{nm})\end{array}$ & $\begin{array}{l}\text { Resonant } \\
\text { wavelength } \\
(\mathrm{nm})\end{array}$ & $\begin{array}{l}\text { Lower } \\
\text { level }\end{array}$ & $\begin{array}{l}\text { Upper } \\
\text { level }\end{array}$ & $\begin{array}{l}\text { Paschen } \\
\text { notation }\end{array}$ & $\begin{array}{l}E_{\mathrm{l}} \\
\left(\mathrm{cm}^{-1}\right)\end{array}$ & $\begin{array}{l}E_{\mathrm{u}} \\
\left(\mathrm{cm}^{-1}\right)\end{array}$ \\
\hline 750.39 & 104.82 & $4 s^{\prime}[1 / 2]_{1}$ & $4 p^{\prime}[1 / 2]_{0}$ & $1 s_{2}$ & 95400 & $\begin{array}{l}\text { Oscillator } \\
\text { strength }\end{array}$ \\
751.47 & 106.67 & $4 s[3 / 2]_{1}$ & $4 p[1 / 2]_{0}$ & $1 s_{4}$ & 93751 & 108723 \\
763.51 & & $4 s[3 / 2]_{2}$ & $4 p[3 / 2]_{2}$ & $1 s_{5}$ & 93144 & 106238 & $0.125^{\mathrm{a}}, 0.2629^{\mathrm{b}}$ \\
794.82 & & $4 s^{\prime}[1 / 2]_{0}$ & $4 p^{\prime}[3 / 2]_{1}$ & $1 s_{3}$ & 94554 & $0.114^{\mathrm{a}}, 0.0675^{\mathrm{b}}$ \\
\hline \hline
\end{tabular}

a Oscillator strength of the observed transition.

$\mathrm{b}$ Oscillator strength of the resonant transition.

can be regarded as a normalized Voigt function, for which the Gaussian component is due to Doppler broadening. The corresponding Doppler-broadened full width at half maximum (FWHM) is given by

$$
\Delta \lambda_{\mathrm{D}}(T)=\lambda_{0} \times 7.16 \times 10^{-7} \times \sqrt{\frac{T}{M}},
$$

where $M$ is atomic mass of the collider. The Lorentzian component arises because of collisions between the excited $\mathrm{Ar}$ atom and a ground state $\mathrm{Ar}$ atom. For lines ending in metastable states, which are not optically connected to the ground state, broadening is mainly by van der Waals interactions, while for the lines ending in the resonant states, additionally, resonance broadening needs to be considered. The analysis of both type of line profiles is discussed below.

The FWHM of the van der Waals broadening, which arises due to the dipole interaction of an excited $\mathrm{Ar}$ atom with the induced dipole in the ground state $\mathrm{Ar}$ (or $\mathrm{He}$ ) atom, can be expressed as ${ }^{52}$

$$
\Delta \lambda_{\mathrm{vdW}}[\mathrm{nm}]=3.0 \times 10^{-15} \lambda^{2}\left(\Delta C_{6}\right)^{2 / 5}\left(\frac{T}{\mu_{\mathrm{P}}}\right)^{3 / 10} N_{\mathrm{P}},
$$

where $\lambda$ is the wavelength of the observed transition $(\mathrm{nm})$, $\Delta C_{6}$ the Leonnard-Jones potential interaction constant, and $\mu_{\mathrm{P}}$ the radiator-perturber reduced mass. For $\mathrm{He}-\mathrm{Ar}$ and $\mathrm{Ar}-\mathrm{Ar}$ interactions, the values of $\mu_{\mathrm{P}}$ are $3.638 \mathrm{amu}$ and $19.97 \mathrm{amu}$, respectively. $N_{\mathrm{P}}$ is the ground-state perturber density $\left(\mathrm{cm}^{-3}\right)$. The value of $\Delta C_{6}$ can be estimated from the following equation

$$
\Delta C_{6}=9.8 \times 10^{-10} \alpha_{\mathrm{P}}\left(\left\langle R_{\mathrm{u}}^{2}\right\rangle-\left\langle R_{1}^{2}\right\rangle\right),
$$

where $\alpha_{\mathrm{P}}$ is the dipole polarizability of the perturber atoms $\left(\mathrm{cm}^{3}\right)$ in their ground states and has values of $2.01 \times 10^{-25}$ $\mathrm{cm}^{3}$ for $\mathrm{He}$ and $1.654 \times 10^{-24} \mathrm{~cm}^{3}$ for $\mathrm{Ar}^{52}\left\langle R_{\mathrm{i}}^{2}\right\rangle$ is the mean square radius of the radiator atom in the upper and lower states of the observed transition of the perturbed atom (expressed in units of Bohr radius $\left.a_{0}\right)$. $\left\langle R_{\mathrm{i}}^{2}\right\rangle$ can be determined from Unsold's hydrogenic approximation as ${ }^{53}$

$$
\left\langle R_{\mathrm{i}}^{2}\right\rangle=\frac{1}{2} n_{\mathrm{i}}^{2}\left[5 n_{\mathrm{i}}^{2}+1-3 l_{\mathrm{i}}\left(l_{\mathrm{i}}+1\right)\right],
$$

where $l_{\mathrm{i}}$ is the active electron orbital quantum number ( 0 for $s$ and 1 for $p$ ) and $n_{\mathrm{i}}^{2}$ is the effective principal quantum number of the sate, given by

$$
n_{\mathrm{i}}^{2}=\frac{E_{\mathrm{ion}}^{\mathrm{H}}}{E_{\mathrm{ion}}^{\mathrm{Ar}}-E_{\mathrm{i}}},
$$

where $E_{\mathrm{ion}}^{\mathrm{H}}$ is the ionization potential of the hydrogen atom or Rydberg constant, $E_{\mathrm{ion}}^{\mathrm{Ar}}$ the ionization potential of the radiator atom (Ar in this case) and $E_{\mathrm{i}}$ the energy of the considered state of the perturbed atom to which the observed transition belongs. Thus, for different concentrations of $\mathrm{Ar}$ in $\mathrm{He}-\mathrm{Ar}$ plasma, $\Delta \lambda_{\mathrm{vdW}}$ could be calculated with $\mathrm{He}$ and $\mathrm{Ar}$ as the perturber atoms. The total width of the vdW broadening is the sum of the individual width perturbed by $\mathrm{He}$ and Ar.

For resonance broadening, the FWHM (in $\mathrm{nm}$ ) can be calculated as $^{52}$

$$
\begin{aligned}
\Delta \lambda_{\mathrm{R}}= & 8.6 \times 10^{-28}\left(\frac{g_{0}}{g_{1}}\right)^{1 / 2} \lambda^{2} \lambda_{\mathrm{R}} f_{\mathrm{lu}} N_{\mathrm{Ar}} \\
= & \chi_{\mathrm{Ar}} R_{\mathrm{Ar}}\left(\frac{300}{T}\right) p,
\end{aligned}
$$

where $\lambda$ and $\lambda_{\mathrm{R}}$ are the wavelengths of the observed and the resonance lines in $\mathrm{nm}$, respectively, $f_{\mathrm{lu}}$ is the oscillator strength of the resonance line starting from the lower state of the transition (given in Table II), $g_{1}=3$ and $g_{0}=1$, the statistical weights of the lower level of the transition and of the ground state, respectively. $N_{\mathrm{Ar}}, \chi_{\mathrm{Ar}}, R_{\mathrm{Ar}}$ and $p$ are the argon ground state number density $\left(\mathrm{cm}^{-3}\right)$, the fraction of Ar in the gas mixture, a constant for resonance broadening of Ar, and the pressure in bar, respectively.

The Stark broadening for the investigated experimental conditions is negligible due to the low electron density in such RF discharges.

The vdW broadening calculations are carried out only for lines ending in the metastable states as lower level $\left(1 s_{3}\right.$ and $1 s_{5}$ ). Since the resonance broadening dominates for $1 s_{2}$ and $1 s_{4}$ states, the vdW broadening has been neglected for these lines. The perturbation due to $\mathrm{He}$ and Ar are considered separately in determining the vdW broadening with Eq. 16,

$$
\Delta \lambda_{\mathrm{vdW}}[\mathrm{nm}]=\chi_{\mathrm{He}} K_{\mathrm{He}}\left(\frac{300}{T}\right)^{0.7}+\chi_{\mathrm{Ar}} K_{\mathrm{Ar}}\left(\frac{300}{T}\right)^{0.7}
$$



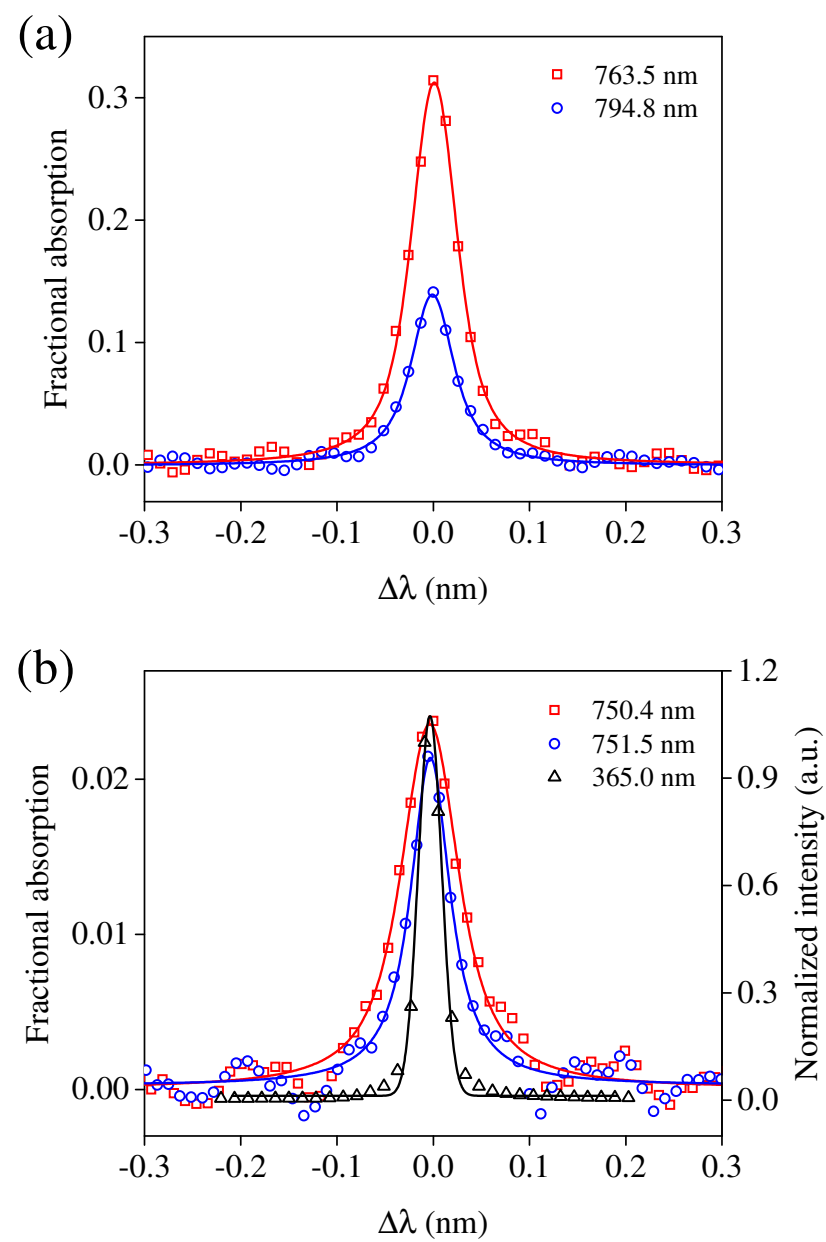

FIG. 6. Absorption profiles of atoms in (a) metastable states (763.5 and $794.8 \mathrm{~nm}$ ), and (b) resonant states (750.4 and $751.5 \mathrm{~nm}$ ) of Ar corresponding to $\mathrm{He}+17 \%$ Ar plasma. The profiles are recorded at the sheath edge, $250 \mu \mathrm{m}$ from the powered electrode. As a comparison, the slit function of the monochromator as determined by the normalized intensity of $\mathrm{Hg}$ I line recorded at $365 \mathrm{~nm}$ is also shown in (b).

$\chi_{\mathrm{He}}$ is the fraction of $\mathrm{He}$ and $\chi_{\mathrm{He}}+\chi_{\mathrm{Ar}}=1 . K_{\mathrm{He}}$ and $K_{\mathrm{Ar}}$ are constants for vdW broadening. Table III compiles the values of these constants. However, as one can see from absorption profiles presented in Fig. 6, their noise level is too high to permit a correct determination of the broadening parameters. We, thus, have used the line profiles of the emission spectra $P(\lambda)$ recorded for the broadband absorption spectroscopy measurements, which have much higher signal/noise ratios (see Fig. 11 in Section III B), for the determination of the pressure broadening constants of the lines reported in Table III.

The convolution of the Gaussian FWHM $\left(\Delta \lambda_{\mathrm{D}}\right)$ and the Lorentzian FWHM $\left(\Delta \lambda_{\mathrm{L}}\right)$ profiles provides the Voigt function to be used as the normalized $\varphi(\lambda)$ in Eq. 14. For a fractional absorption of only a few $\%, W$ can be directly deduced by a linear approximation of the term $(1-\exp (-\sigma(\lambda, T) \cdot n \cdot l))$ in Eq. 13 to $\sigma(\lambda, T) \cdot n \cdot l$, and thus the density, $n$ can be directly determined using equations Eqs. 13-20. However, for
TABLE III. Measured van der Waals and resonance broadening constants of used Ar lines scaled for atmospheric pressure and $300 \mathrm{~K}$ in pure $\mathrm{Ar}$ and $\mathrm{He}$.

\begin{tabular}{lccc}
\hline \hline State & $\begin{array}{c}K_{\mathrm{He}} \\
(\mathrm{nm})\end{array}$ & $\begin{array}{c}K_{\mathrm{Ar}} \\
(\mathrm{nm})\end{array}$ & $\begin{array}{c}R_{\mathrm{Ar}} \\
(\mathrm{nm})\end{array}$ \\
\hline $1 s_{3}$ & 0.023 & 0.032 & - \\
$1 s_{5}$ & 0.020 & 0.028 & - \\
$1 s_{2}$ & - & - & 0.189 \\
$1 s_{4}$ & - & - & 0.050 \\
\hline \hline
\end{tabular}

strongly absorbing lines, due to the non-linearity of $W$ as a function of $n$, there is no analytical expression to relate these two parameters. Therefore, a simulation was performed to deduce $W$ for different values of $n .^{31,49}$ The experimentally measured values of $W$ were compared with those of simulated $W$ to determine the absolute time-averaged and line-of-sight integrated densities of atoms in $1 s_{2}, 1 s_{3}, 1 s_{4}$ and $1 s_{5}$ states of Ar. The reported uncertainties on the estimated densities were determined from twice the standard deviation based on the uncertainties in the fitting of the Voigt profile to the absorption line profile to determine $W$ and the asymmetry in the spatially distributed density profile. The uncertainties due to the estimated error in the gas temperature determination were negligible. Moreover, there exists uncertainty in the spatial variation of the experimental data. For instance, spatial variations of $\sim 20 \%$ were observed in the density profiles of the $1 s_{3}$ and $1 s_{5}$ metastable states, which could be increased to $45 \%$ close to the detection limit, in pure Ar discharge.

\section{H. Resonance radiation transport modeled by Monte Carlo simulation}

To explain the measured profile of atoms in resonance states in the discharge, we implemented a resonance radiation transport model. We considered the argon lines $3 s^{2} 3 p^{6}-$ $3 s^{2} 3 p^{5}\left({ }^{2} \mathrm{P}_{1 / 2}^{0}\right) 4 s(104.8 \mathrm{~nm})$ and $3 s^{2} 3 p^{6}-3 s^{2} 3 p^{5}\left({ }^{2} \mathrm{P}_{3 / 2}^{0}\right) 4 s$ $(106.7 \mathrm{~nm})$ in an optically thick slab corresponding to the plasma volume within $2 \mathrm{~mm}$ gap between the electrodes. The density of photo-excited atoms has been estimated using a kinetic Monte Carlo method; details can be found in ${ }^{54-56}$. The inter-electrode gap is divided into 100 slabs of $20 \mu \mathrm{m}$ thickness each, which form the grid points for the Monte Carlo simulation. The $20 \mu \mathrm{m}$ thickness of the slabs enables to resolve the $50 \mu \mathrm{m}$ spatial resolution of the experiment. ${ }^{31}$ The two slabs at a distance of $0.4 \mathrm{~mm}$ from the walls corresponding to the locations where the maximum densities of atoms in the resonant states were measured (see further) are taken to be the source of the initial photons for this simulation. In this method, random walks for photons are generated numerically and they serve in the evaluation of the number of absorption processes that occur at a given location $z$ in each slab. At each walk, a photon "birth" is first performed through random generation of the position $\mathbf{r}$, the wavelength $\lambda$, and the direction $\mathbf{n}=\mathbf{k} /|\mathbf{k}|$. The angles $\theta$ and $\phi$ relative to the direction $\mathbf{n}$ (expressed using the spherical coordinates) are gener- 
ated using a uniform distribution for $\cos \theta \equiv \mu$ and for $\phi$. The wavelength $\lambda$ is generated assuming a Lorentzian distribution, $\phi(\lambda)$, accounting for collisional broadening. Once $z, \theta, \phi$ and $\lambda$ have been generated, the path length $s$ covered by the photon before its absorption by an Ar atom is generated using an exponential distribution, of parameter $a(\lambda)$, the mean free path of the photon, defined as $a(\lambda)=1 /([\mathrm{Ar}] \cdot \sigma(\lambda))$, where [Ar] is the density of argon atoms and $\sigma(\lambda)$ is the absorption cross section, given in Eq. 14 , but with $\lambda_{0}$ and $f$ relative to the resonance transition. Note that the photon path length $s$ is wavelength dependent. The absorption occurs at the coordinate $\mathbf{r}^{\prime}=\mathbf{r}+\mathbf{n} s$. During the lifetime of the newly excited atom, the proportion of emission of a new photon is estimated using the branching ratio $A_{\mathrm{ul}} /\left(A_{\mathrm{ul}}+C_{\mathrm{ul}}\right)$ where $A_{\mathrm{ul}}$ is the Einstein coefficient for spontaneous emission and $C_{\mathrm{ul}}$ is the collisional de-excitation frequency. A uniform random variable in the $[0,1]$ interval, $u$, is generated and provides the process that occurs (photon emission if $u<A_{\mathrm{ul}} /\left(A_{\mathrm{ul}}+C_{\mathrm{ul}}\right)$; quenching otherwise) at the location $\mathbf{r}^{\prime}$. In the case where a photon emission process occurs, new values are generated for the direction $\mathbf{n}$, the wavelength $\lambda$, and the path length $s$, according to the procedure indicated above. In the quenching case, the random walk ends. The simulation ends once a given number of random walks has been performed. The calculation yields the photo-excitation rate $W_{\mathrm{lu}}\left(\mathrm{s}^{-1}\right)$ at the $z$ location within the 100 grid points. The total number of initially generated photon + photon absorption occurring at each grid point is evaluated using a counter. This quantity is representative of the density of atoms in the resonance state. About $10^{6}$ initially generated photons, followed by random walks, was necessary in order to have a reasonable $(>100)$ signal/noise ratio on calculated densities.

\section{RESULTS AND DISCUSSIONS}

\section{A. Determination of $T_{e}$ and $n_{e}$}

Fig. 1(b)-(d) present emission images of plasmas in $\mathrm{Ar}$, $\mathrm{He}$ and $\mathrm{He}+17 \%$ Ar, respectively. The intense bright "white" emission in the bulk of the pure Ar discharge shown in Fig. 1(b) suggests the presence of continuum radiation in the visible wavelengths. The measured time and spatially averaged absolute emission spectra of the RF-driven capacitive discharges in $\mathrm{Ar}$ and $\mathrm{He}$ and the fitted theoretical spectra are shown in Fig. 7. This measured emission spectrum is fitted with the calculated emissivity of the neutral bremsstrahlung radiation $\left(\varepsilon_{\mathrm{ea}}\right)$ following the procedure described in Section II B. The fit was performed by matching the lowest intensity features of the spectra in their high wavelength sides. For argon, the fit suggests the presence in the $450-650 \mathrm{~nm}$ range of emission having another origin.

The neutral Bremsstrahlung $\varepsilon_{\text {ea }}$ is calculated by using both Maxwellian and non-Maxwellian EEDFs via the Boltzmann solver Bolsig ${ }^{38}$ (cross-section obtained from ${ }^{40,57}$ ) as the EEDF deviates significantly from a Maxwell-Boltzmann distribution. ${ }^{58,59}$ The obtained electron properties $\left(T_{\mathrm{e}}\right.$ and $n_{\mathrm{e}}$ ) are shown in Table IV. For the calculation of the non-
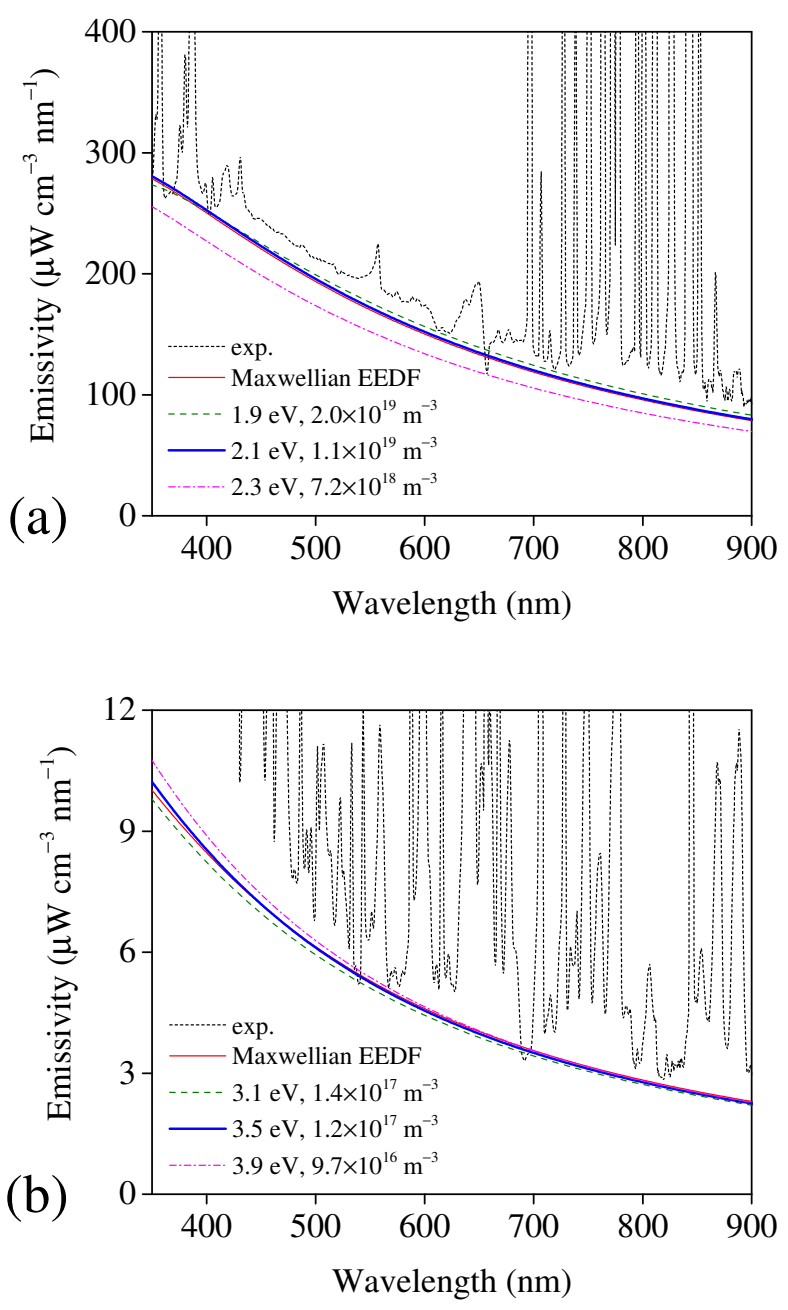

FIG. 7. The measured absolute intensity of RF plasma emission in (a) $\mathrm{Ar}$ at a discharge power of $13.5 \mathrm{~W}$ and (b) $\mathrm{He}$ at a discharge power of $14.0 \mathrm{~W}$, and gas flow rate of $1 \mathrm{slm}$ fitted with the calculated emissivities of the e-a bremsstrahlung continuum radiation using both Maxwellian and non-Maxwellian EEDFs. The obtained values for $\mathrm{Ar}$ and $\mathrm{He}$ plasmas are reported in Table IV.

Maxwellian EEDF, an ionization degree of $10^{-7}$ was used. The uncertainty in the absolute calibration due to day-to-day variation in the intensity of plasma and halogen lamp emissions was $\sim 12 \%$, while the random variation in the absolute intensity was $\sim 14 \%$ for helium and $\sim 12 \%$ for argon. The random uncertainty due to the experimental variation between different measurements were negligible as compared to the aforementioned uncertainties. The final uncertainty in the electron density was estimated by using the root mean square error. While the relative calibration is more accurate than the absolute calibration, the uncertainty in $n_{\mathrm{e}}$ also leads to additional uncertainty in $T_{\mathrm{e}}$. The fit resulted in an effective $T_{\mathrm{e}}$ of $2.1 \pm 0.3 \mathrm{eV}$ for argon and $3.5 \pm 0.4 \mathrm{eV}$ for helium. The fit also yielded an $n_{\mathrm{e}}$ of $(1.1 \pm 0.7) \times 10^{19} \mathrm{~m}^{-3}$ for argon and $(1.2 \pm 0.2) \times 10^{17} \mathrm{~m}^{-3}$ for helium. However, a Maxwellian distribution led to a significantly lower $T_{\mathrm{e}}$ for both Ar and He, 
with an $n_{\mathrm{e}}$, which is $\sim 5$ times higher to obtain the same profile, particularly for Ar (Table IV). This suggests that it is important to consider non-Maxwellian effects in the EEDF to obtain accurate $T_{\mathrm{e}}$ and $n_{\mathrm{e}}$ from neutral bremsstrahlung measurements.

An alternative and independent approach to measure the electron density is using plasma impedance measurements, as described in Section II D. The voltage and current waveforms for Ar plasma operating at a discharge power of $13.5 \mathrm{~W}$ with an applied peak-to-peak voltage of $853 \mathrm{~V}$ and a current of 1.3 A are shown in Fig. 8. The sheath width calculated for these parameters from the equivalent electrical model described in Section II D is $180 \mu \mathrm{m}$. Additionally, the sheath width was obtained from optical images of the discharge at the same operating conditions. The digital images were converted to spatially-distributed intensity profiles by binning the pixels in the vertical direction (not shown here) and then normalized with respect to the maximum intensity for better visualization. These normalized total intensity profiles for discharge in $\mathrm{Ar}$ and He are shown in Fig. 9. The apparent peaks at positions 0 (grounded electrode) and $2 \mathrm{~mm}$ (powered electrode) are most likely due to the grazing-angle reflections of the plasma emission by electrodes. Finally, the sheath width was determined by the difference in the number of pixels when the intensity drops to $61 \%$ of the intensity at the sheath edge..$^{30,45}$ The sheath edge is marked by a horizontal gray dashed line in Fig. 9. This yielded a sheath width of $170 \mu \mathrm{m}$ in pure argon discharge, which is very similar to that determined from the electrical model within the accuracy of the experiment. Using Eq. 10 and Eq. 11, the $n_{\mathrm{e}}$ is calculated to be $1.3 \times 10^{18} \mathrm{~m}^{-3}$, which is an order of magnitude lower than the $n_{\mathrm{e}}$ determined by neutral Bremsstrahlung assuming a non-Maxwellian distribution. The same procedure was followed to obtain $s_{0}=380$ $\mu \mathrm{m}$ and $n_{\mathrm{e}}=1.8 \times 10^{17} \mathrm{~m}^{-3}$ for He. The electron density is of the same order of magnitude as obtained from the neutral Bremsstrahlung. However, the sheath width obtained from the optical images was found to be $160 \mu \mathrm{m}$, which is much smaller than that obtained from the electrical model. A summary of the sheath widths and electron densities as calculated from the equivalent electrical model for $\mathrm{Ar}$ and $\mathrm{He} \mathrm{RF}$ glow discharges as functions of discharge power are shown in Fig. 10. Similar trends and values are obtained for the RF COST reference microplasma jet operating in the abnormal or $\alpha$-mode with an inter-electrode gap distance of $1 \mathrm{~mm} .{ }^{60}$

An independent estimate of $T_{\mathrm{e}}$ can be obtained from the power balance. Assuming momentum transfer from electrons to neutrals by elastic collisions as the dominant loss mechanism, the input electrical power density, $P / V\left[\mathrm{~W} \mathrm{~m}^{-3}\right]$ can be written as follows 44

$$
\frac{P}{V}=n_{\mathrm{e}} n_{\mathrm{a}} k_{\mathrm{el}}\left(T_{\mathrm{e}}\right) \frac{3 m_{\mathrm{e}}}{m_{\mathrm{Ar}}} k_{\mathrm{B}}\left(T_{\mathrm{e}}-T\right),
$$

where $k_{\mathrm{el}}$ is the rate constant for elastic electron scattering on Ar calculated by Bolsig+. ${ }^{61}$ The ionization and excitation rate constants are neglected in Eq. 22 as these are several orders of magnitude lower than $k_{\mathrm{el}}$ in the investigated operating conditions. Using Eq. 22, at $P / V=37.2 \mathrm{~W} \mathrm{~cm}^{-3}$ assuming

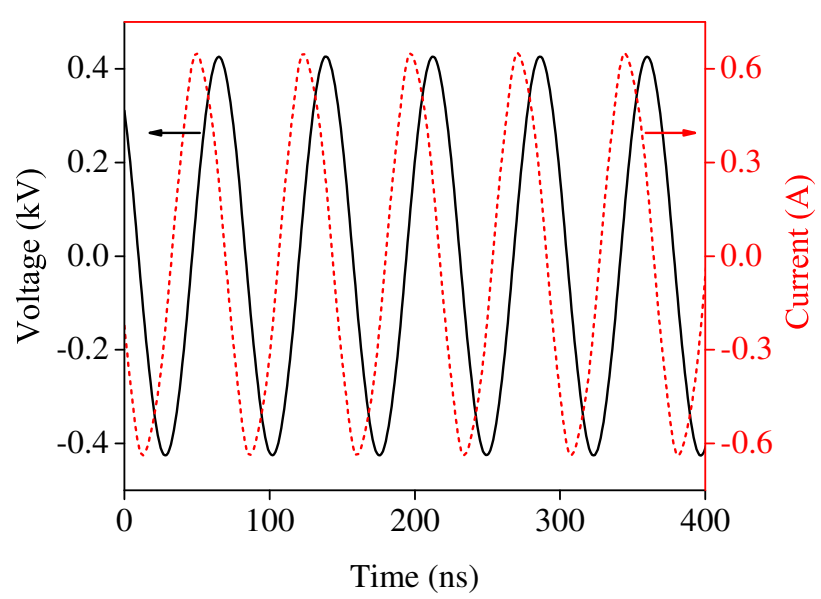

FIG. 8. Voltage and current waveforms of the Ar RF glow discharge.

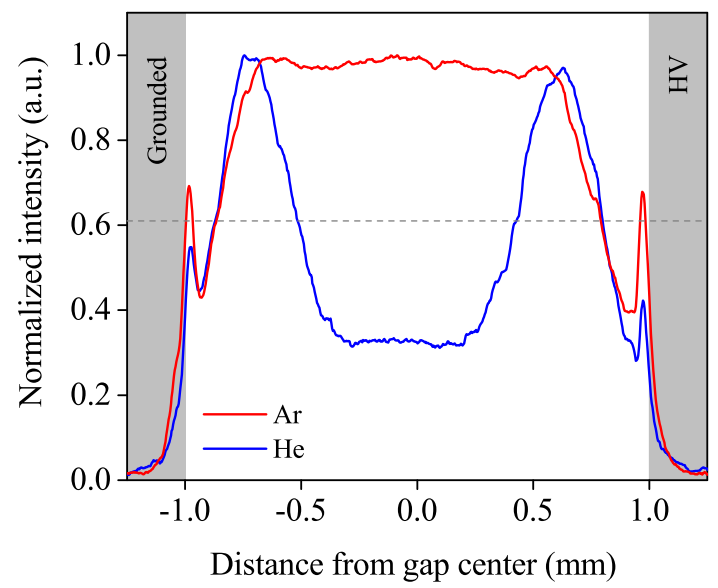

FIG. 9. Normalized total emission intensity profiles of Ar and He RF glow discharges at discharge powers of $13.5 \mathrm{~W}$ and $14.0 \mathrm{~W}$, respectively. The shaded regions represent the location of the grounded and the high-voltage (HV) electrodes.

$V$ is equal to the entire plasma volume, $T_{\mathrm{e}}$ was evaluated at the same $n_{\mathrm{e}}$ as obtained from continuum emission fitting approach and found to be $1.2 \mathrm{eV}$ for Ar. Similarly, assuming a $T_{\mathrm{e}}$ of $2.1 \mathrm{eV}$, a mean electron density of $2.2 \times 10^{18} \mathrm{~m}^{-3}$ was obtained. The same approach was applied to the He case and the results are shown in Table IV. The obtained electron temperature from the power balance in $\mathrm{Ar}(1.2 \mathrm{eV})$ is much lower than that obtained from the continuum radiation approach. While the power approach is an approximation, a possible explanation for the higher $T_{\mathrm{e}}$ obtained from the continuum radiation measurement is the contribution of a higher $T_{\mathrm{e}}$ in the near sheath region due to the non-local electron kinetics effects. Indeed, particularly in Ar, the length scale of electron energy relaxation at atmospheric pressure as given by ${ }^{62}$

$$
\lambda_{\varepsilon}=\frac{\lambda_{\mathrm{e}}}{\sqrt{\delta}}
$$




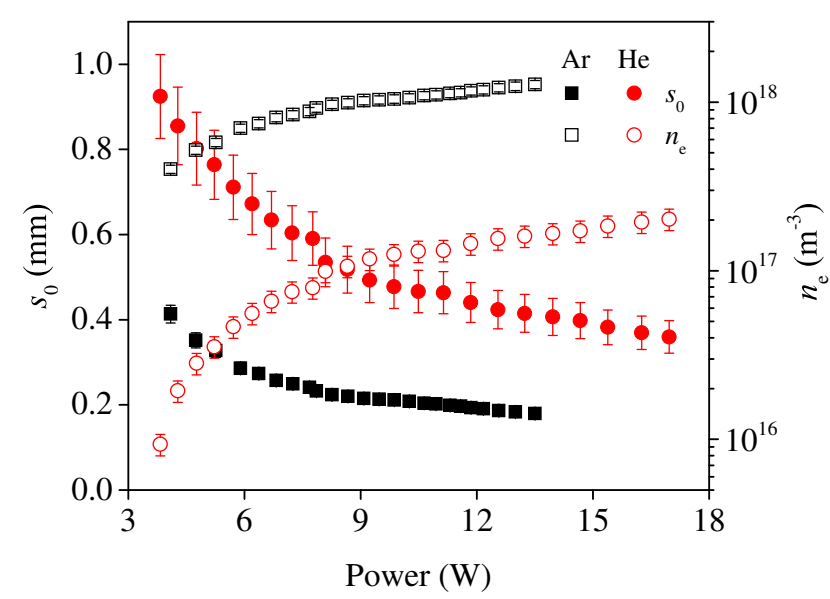

FIG. 10. Time-averaged sheath width and electron density as a function of the discharge power for Ar and He RF glow discharges obtained using Eq. 10 and Eq. 11.

is large ( $\sim 240 \mu \mathrm{m})$. In Eq. $23, \lambda_{\mathrm{e}}$ is the mean free path of electron in $\operatorname{Ar}$ and $\delta=2 m_{\mathrm{e}} / m_{\mathrm{Ar}}$. Hence, a significant part of the inter-electrode gap can contain electrons with a much higher energy than estimated from a bulk local field approximation analysis. In addition, the power dissipated in the sheath is unknown and not accounted for, which leads to additional uncertainties.

For a similar geometry with $2 \mathrm{~mm}$ inter-electrode gap, a driving frequency of $13.56 \mathrm{MHz}$ and similar power density in $\mathrm{Ar}$, the modeling results reported an $n_{\mathrm{e}}$ of $8 \times 10^{17} \mathrm{~m}^{-3}$ in the bulk and a $T_{\mathrm{e}}$ of $\sim 2.5 \mathrm{eV} .{ }^{63}$ This model accounted for the deviations from Maxwellian distribution by calculating the EEDF from Bolsig+ and also included stepwise ionization as one of the prominent electron production mechanisms in the plasma. Using similar model, but with a different geometry, an $n_{\mathrm{e}}$ of $7 \times 10^{17} \mathrm{~m}^{-3}$ was reported, while the $T_{\mathrm{e}}$ varied from $2.1 \mathrm{eV}$ to $2.9 \mathrm{eV}$ within one RF discharge cycle. ${ }^{64} \mathrm{Al}-$ though the calculated $n_{\mathrm{e}}$ in these models is lower than that determined in the current study, the space-averaged $T_{\mathrm{e}}$ is similar. An experimental study on Ar and He RF discharges at atmospheric pressure used the current density and a steadystate power balance to determine $n_{\mathrm{e}}$ and $T_{\mathrm{e}} .{ }^{65}$ Although the reported $T_{\mathrm{e}}$ values for both $\mathrm{Ar}$ and He plasmas are lower (1.1 $\mathrm{eV})$ than that determined in the current study, $n_{\mathrm{e}}$ value for Ar plasma $\left((2.4 \pm 0.5) \times 10^{18} \mathrm{~m}^{-3}\right)$ is of the same order of magnitude as estimated in this study within the experimental error, while it is almost an order of magnitude larger in $\mathrm{He}$ $\left((9.3 \pm 1.8) \times 10^{17} \mathrm{~m}^{-3}\right)$. A one-dimensional fluid model applied to an Ar RF discharge operating at atmospheric pressure using a similar electrical model analysis as ${ }^{65}$ reported an $n_{\mathrm{e}}$ of $\sim 2 \times 10^{18} \mathrm{~m}^{-3}$, ${ }^{66}$ which is 5.5 times smaller than the value estimated in this study. A self-consistent computational study of a He RF discharge reported an $n_{\mathrm{e}}$ of $3.6 \times 10^{17} \mathrm{~m}^{-3}$, only thrice the value determined in the current study. ${ }^{67}$

Thus, from these reported results, it is evident that different methods (continuum radiation, electrical model and power balance) yield different values of $n_{\mathrm{e}}$ and $T_{\mathrm{e}}$. While both elec-
TABLE IV. Comparison of electron properties $\left(T_{\mathrm{e}}\right.$ and $\left.n_{\mathrm{e}}\right)$ of RFdriven capacitive Ar and He glow discharges at atmospheric pressure with a discharge power of $13.5 \mathrm{~W}$ and $14.0 \mathrm{~W}$, respectively obtained from the continuum radiation $\varepsilon_{\text {ea }}$ fitting with the assumption of a Maxwellian EEDF and non-Maxwellian EEDF.

\begin{tabular}{lcccc}
\hline \hline & \multicolumn{2}{c}{$\mathrm{Ar}$} & \multicolumn{2}{l}{$\mathrm{He}$} \\
Method & $\begin{array}{c}T_{\mathrm{e}} \\
(\mathrm{eV})\end{array}$ & $\begin{array}{c}n_{\mathrm{e}} \\
\left(\mathrm{m}^{-3}\right)\end{array}$ & $\begin{array}{c}T_{\mathrm{e}} \\
(\mathrm{eV})\end{array}$ & $\begin{array}{c}n_{\mathrm{e}} \\
\left(\mathrm{m}^{-3}\right)\end{array}$ \\
\hline Continuum radiation & & & & \\
$\quad$ Maxwellian EEDF & 1.1 & $5.3 \times 10^{19}$ & 2.5 & $2.0 \times 10^{17}$ \\
$\quad$ Non-Maxwellian EEDF & 2.1 & $1.1 \times 10^{19}$ & 3.5 & $1.2 \times 10^{17}$ \\
Electrical model & - & $1.3 \times 10^{18}$ & - & $1.8 \times 10^{17}$ \\
Power balance & 1.2 & $\left(1.1 \times 10^{19}\right)$ & 3.1 & $\left(1.2 \times 10^{17}\right)$ \\
\hline \hline
\end{tabular}

trical model and power balance methods have assumptions, and, in particular for He, which do not account for important impact of impurities leading to Penning ionization, ${ }^{68,69}$ the only uncertainty in the continuum radiation approach is mainly due to the fitting and absolute calibration of the continuum emission intensity. Thus, the continuum radiation approach is believed to be the most accurate.

In summary, the time and space-averaged $T_{\mathrm{e}}$ and $n_{\mathrm{e}}$ values determined from the continuum emission of the argon plasma are $2.1 \pm 0.3 \mathrm{eV}$ and $(1.1 \pm 0.7) \times 10^{19} \mathrm{~m}^{-3}$, respectively, while for helium plasma, a $T_{\mathrm{e}}$ of $3.5 \pm 0.4 \mathrm{eV}$ and an $n_{\mathrm{e}}$ of $(1.2 \pm 0.2) \times 10^{17} \mathrm{~m}^{-3}$ are determined.

\section{B. Measured Ar line widths}

The collisional broadenings, or the Lorentzian widths of $\mathrm{Ar}$ lines used in this study for the measurement of $\operatorname{Ar}(1 s)$ atoms density in both metastable and resonant states, were determined from the plasma induced emission spectra, as shown in Fig. 11 for the $750 \mathrm{~nm}$ spectral region, recorded in pure argon plasma. The gas temperature, obtained from the rotational temperature of $\mathrm{OH}(A-X)$ emission (see Section II F), is $365 \mathrm{~K}$ in this plasma. Fitting the profiles of these lines with Voigt functions provides their Gaussian and Lorentzian widths. In the example of Fig. 11, the best fit of the $751.5 \mathrm{~nm}$ line is obtained with $w_{\mathrm{G}}=2.27 \pm 0.38$ pixels and $w_{\mathrm{L}}=4.17 \pm 0.26$ pixels, for the Gaussian and Lorentzian widths, respectively. Uncertainties are twice the standard deviations from the fit. With the $11.47 \mathrm{pm} /$ pixel linear dispersion of the spectrometer, deduced from the numbers of pixels between the positions of 750.4 and $751.5 \mathrm{~nm}$ lines, the widths in pixel are converted to $\Delta \lambda_{\mathrm{G}}=26 \pm 4 \mathrm{pm}$ and $\Delta \lambda_{\mathrm{L}}=48 \pm 3 \mathrm{pm}$. Given that at $365 \mathrm{~K}$ the Doppler widths of the $750 \mathrm{~nm}$ lines are only about $1.6 \mathrm{pm}$, we can conclude that $\Delta \lambda_{\mathrm{D}}$ can be neglected and $\Delta \lambda_{\mathrm{G}}=26 \pm 4$ pm corresponds to the instrumental width. This apparatus width is slightly smaller than the $29 \mathrm{pm}$ deduced from the $365 \mathrm{~nm} \mathrm{Hg}$ line width, as shown in Fig. 6(b). The difference is in accordance with the reduction of the linear dispersion of the spectrometer with increasing rotation angle of the grating, thus with the wavelength. Fixing the same instrumental 


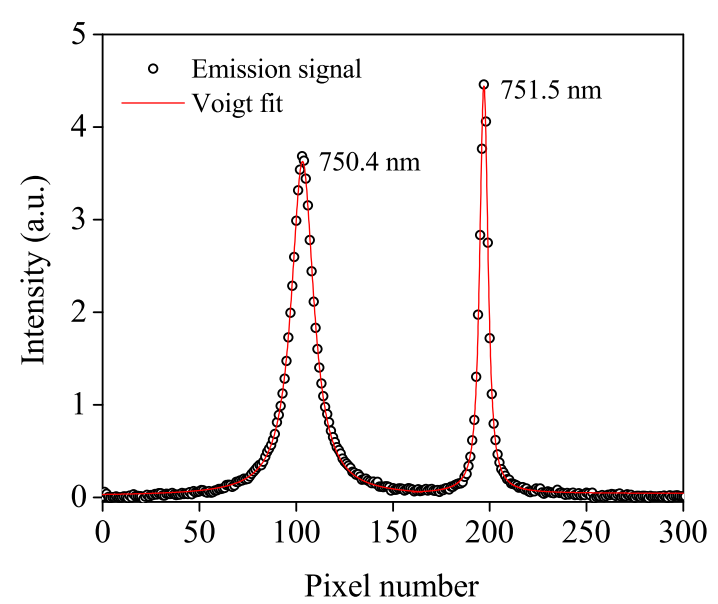

FIG. 11. Experimentally recorded emission spectra of $\operatorname{Ar}(1 s)$ lines in the resonant states fitted with a Voigt profile for pure Ar discharge operating at $13.5 \mathrm{~W}$ and measured $250 \mu \mathrm{m}$ away from the electrode surface.

width $w_{\mathrm{G}}=2.27$ pixels for the fit of the $750.4 \mathrm{~nm}$ line, we obtain the Lorentzian width of the line $w_{\mathrm{L}}=13.5 \pm 0.2$ pixels, corresponding to $\Delta \lambda_{\mathrm{L}}=155 \pm 3 \mathrm{pm}$. From the above deduced $w_{\mathrm{L}}$ values in pure Ar plasma at $T_{\mathrm{g}}=365 \mathrm{~K}$, the broadening coefficients at "standard conditions" of 1 atmosphere and $300 \mathrm{~K}$ would be $58 \pm 4$ and $189 \pm 4 \mathrm{pm}$ for $751.5 \mathrm{~nm}$ and $750.4 \mathrm{~nm}$ lines, respectively. Following the same procedure, the Lorentzian widths of the $763.5 \mathrm{~nm}$ and $794.8 \mathrm{~nm}$ lines have been deduced from the Voigt fits of their emission spectra (not shown) recorded in pure argon plasma. With fixed Gaussian width of $w_{\mathrm{G}}=2.27$ pixels, the deduced Lorentzian widths are $2.68 \pm 0.08$ and $2.77 \pm 0.10$ pixels for 794.8 and $763.5 \mathrm{~nm}$ lines, respectively. Taking into account the uncertainty on $w_{\mathrm{G}}=2.27 \pm 0.38$ pixels, the above values lead to $\Delta \lambda_{\mathrm{L}}=30.8 \pm 4.5$ and $31.7 \pm 4.5 \mathrm{pm}$ at $365 \mathrm{~K}$ for these lines. These values correspond to $\Delta \lambda_{\mathrm{L}}=35 \pm 5 \mathrm{pm}$ for the 794.8 $\mathrm{nm}$ line and $\Delta \lambda_{\mathrm{L}}=36 \pm 5 \mathrm{pm}$ for the $763.5 \mathrm{~nm}$ line at 1 atmosphere and $300 \mathrm{~K}$. Our experimental values are compiled in Table V, as well as the theoretical broadening coefficients, deduced from Eq. 20 and Eq. 21, and those reported in the literature. The pressure range and the estimated temperatures, as reported by authors, are also included in this table and, for the sake of clarity, all reported broadening coefficients are converted to their "standard conditions" of 1 atmosphere and 300 $\mathrm{K}$, by using the temperature dependence of $\Delta \lambda_{\mathrm{L}}$, as indicated in Eq. 20 and Eq. 21. The reported uncertainty in column 2 accounts for the uncertainty deduced from the fit plus the $2.7 \%$ uncertainty in the determination of the gas temperature.

The measured broadening coefficient of $750.4 \mathrm{~nm}$ line is in perfect agreement with its theoretical value and very close to the mean value of coefficients reported in ${ }^{70},{ }^{72}$ and $^{74}$. These later have been measured in atmospheric pressure thermal plasmas, in which the plasma is usually not spatially homogeneous and the determination of the correct gas temperature is a hard task and is further complicated by additional Stark broadening of the lines. Our experimental coefficient for the

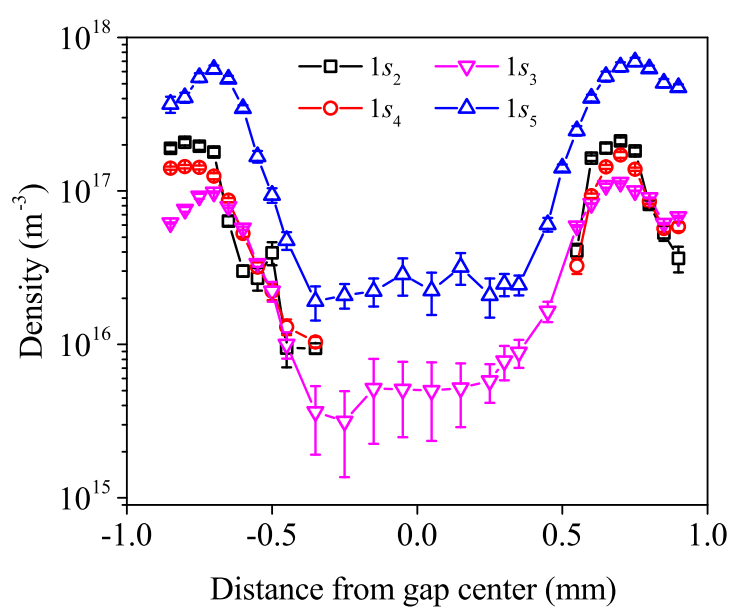

FIG. 12. Time-averaged spatially resolved absolute densities of atoms in $1 s_{2}, 1 s_{3}, 1 s_{4}$ and $1 s_{5}$ states of argon in pure Ar plasma at a discharge power of $13.5 \mathrm{~W}$ and total gas flow rate of $1 \mathrm{slm}$.

$751.5 \mathrm{~nm}$ line being almost $20 \%$ larger compared to the theoretical one, can be justified by a contribution from the van der Waals broadening of the upper and lower states of the 751.5 $\mathrm{nm}$ line. In fact, the theoretical values reported in the $3^{\text {rd }}$ column of Table V for 750.4 and $751.5 \mathrm{~nm}$ lines represent only the resonance broadening of the lower states of these transitions, $1 s_{2}$ and $1 s_{4}$ states, respectively. Values reported in ${ }^{71}$ and $^{72}$ have been obtained from the emission line profiles in glow discharges, analyzed with a Fabry-Perot interferometer. At the low working pressures in these works, the Lorentzian width of the lines was comparable, or even smaller than the Doppler width, introducing large uncertainty on deduced $\Delta \lambda_{\mathrm{L}}$. Reported uncertainties on the broadening coefficient of 751.5 nm line are $\pm 23 \%$ in $^{71}$ and $\pm 18 \%$ in $^{72}$. Our result for the van der Waals broadening of $794.8 \mathrm{~nm}$ line is in perfect agreement with the calculated and previously reported values. But for $763.5 \mathrm{~nm}$ lines, we have measured slightly larger value than the calculated one and those reported in the literature. Note that the uncertainties on these later broadening coefficients are large. However, considering that, with the estimated $n_{\mathrm{e}} \leq 10^{19}$ $\mathrm{m}^{-3}$ (see Table IV) the Stark broadenings of the lines are negligibly small ${ }^{75}$ and the high precision on measured gas temperature, we are reasonably confident on our measured $\Delta \lambda_{\mathrm{L}}$ values reported in the $2^{\text {nd }}$ column of Table $\mathrm{V}$.

\section{Spatial distribution of Ar atoms in metastable and resonant states}

\section{Ar RF glow discharge}

The absolute densities of atoms in metastable $\left(1 s_{3} \& 1 s_{5}\right)$ and resonant $\left(1 s_{2} \& 1 s_{4}\right)$ states are determined following the detailed procedure described in Section II G. Fig. 12 shows the spatial distribution of the absolute densities of these atoms in the inter-electrode gap of pure argon discharge as a function of distance. 
TABLE V. Pressure broadening coefficients by argon of the lines used for the determination of $\operatorname{Ar}(1 s)$ atoms density. For the sake of clarity all $\Delta \lambda_{\mathrm{L}}$ values are for the "standard conditions" of 1 atmosphere at $300 \mathrm{~K}$ and previously reported widths are re-scaled for their temperature dependence by using Eq. 20 and Eq. 21.

\begin{tabular}{|c|c|c|c|c|c|c|c|c|}
\hline $\begin{array}{l}\text { Wavelength } \\
\text { (nm) }\end{array}$ & $\begin{array}{l}\left(\Delta \lambda_{\mathrm{L}}\right)_{\exp } \\
(\mathrm{pm})\end{array}$ & $\begin{array}{c}\left(\Delta \lambda_{\mathrm{L}}\right)_{\text {theory }} \\
(\mathrm{pm})^{\mathrm{a}}\end{array}$ & & & $\left(\Delta \lambda_{\mathrm{L}}\right)_{\mathrm{prev}}$ & eriments & & \\
\hline Ref & This work & & 70 & 53 & 71 & 72 & 73 & 74 \\
\hline $\mathrm{P}(\mathrm{bar})^{\mathrm{b}}$ & 1 & 1 & $0.5-2$ & $\leq 0.02$ & $\leq 0.1$ & $\leq 0.02$ & $1-2.5$ & $1-3$ \\
\hline$T_{\mathrm{g}}(\mathrm{K})^{\mathrm{c}}$ & 365 & 300 & 1130 & 300 & $360-500$ & 300 & 2000 & 3900 \\
\hline $750.4\left(1 s_{2}\right)$ & $189 \pm 9$ & 188 & 213 & - & - & 208 & - & 165 \\
\hline $751.5\left(1 s_{4}\right)$ & $58 \pm 6$ & 49 & 59 & - & 41 & 48 & 75 & 57 \\
\hline $763.5\left(1 s_{5}\right)$ & $36 \pm 6$ & 28 & - & 30 & - & - & 32 & 22 \\
\hline $794.8\left(1 s_{3}\right)$ & $35 \pm 6$ & 32 & 31 & - & - & - & 30 & 20 \\
\hline
\end{tabular}

a From Eq. 20 and Eq. 21

${ }^{\mathrm{b}}$ Gas pressure

${ }^{c}$ Estimated temperature

The density profiles in these states correlate well with the excitation zone in an RF-driven atmospheric pressure discharge operated in $\alpha$-mode. ${ }^{67,76}$ A maximum density of atoms in metastable and resonant states in excess of $10^{17} \mathrm{~m}^{-3}$ are observed near the sheath edge region, 250-300 $\mu \mathrm{m}$ away from the electrodes, while the densities decline towards the electrodes and in the bulk plasma. The absolute densities of $\mathrm{Ar}$ atoms in $1 s_{3}$ and $1 s_{5}$ metastable states are more than an order of magnitude smaller in the bulk plasma than in the sheath region. A similar trend is observed for the absolute densities of $\mathrm{Ar}$ in $1 s_{2}$ and $1 s_{4}$ resonant states, with densities dropping below the detection limit in the bulk of the plasma. The density maxima near the sheath region are due to the elevated $E / N$ in this region, leading to enhanced excitation and ionization rates in this region. ${ }^{3}$ The peak density of atoms in $1 s_{5}$ state is the highest $\left(7 \times 10^{17} \mathrm{~m}^{-3}\right)$, while the lowest density at the same location is that of atoms in $1 s_{3}$, the other metastable state $\left(9.7 \times 10^{16} \mathrm{~m}^{-3}\right)$. The major production mechanism of Ar atoms in the $1 s$ states is electron-impact excitation from the ground state, directly or after radiative cascades from $2 p$ states, following their excitation. ${ }^{21}$ Assuming a Maxwellian EEDF, with $T_{\mathrm{e}}=1.1 \mathrm{eV}$ and $n_{\mathrm{e}}=5.3 \times 10^{19} \mathrm{~m}^{-3}$ obtained from the continuum emission measurement (see Table IV), the production rate could be as high as $6 \times 10^{25} \mathrm{~m}^{-3} \mathrm{~s}^{-1}$ for the $1 s_{5}$ state. ${ }^{18,77}$ However, as the ground-state excitation rate coefficients are highly sensitive to a variation of EEDF, ${ }^{18}$ a nonMaxwellian EEDF strongly impacts this reaction rate, and the production rate will be reduced by more than 3 orders of magnitude, $2.5 \times 10^{22} \mathrm{~m}^{-3} \mathrm{~s}^{-1} \cdot{ }^{38}$ In this case, a non-Maxwellian EEDF increases the likelihood of other dominant reaction mechanisms for the $1 s_{5}$ production. In an atmospheric pressure $\mathrm{Ar}$ glow discharge, $\mathrm{Ar}^{+}$ions produced by ionization are rapidly converted by 3-body reaction involving two Ar atoms, into $\mathrm{Ar}_{2}^{+}$dimer ions, ${ }^{63}$ which rapidly recombine with electrons to produce Ar atoms in excited states; all these excited atoms ending finally in $1 s$ states.

The dominant mechanism for the destruction of $1 s_{5}$ and $1 s_{3}$ metastable atoms is via the three-body collision with the ground-state argon atoms forming argon excimer: ${ }^{78}$

$$
\operatorname{Ar}\left(1 s_{5}\right)+2 \mathrm{Ar} \rightarrow \mathrm{Ar}_{2}^{*}+\mathrm{Ar}
$$

and by the electron collision induced transfer to the $1 s_{4}$ and $1 s_{2}$ resonant states: $:^{2,21,28}$

$$
\mathrm{e}^{-}+\operatorname{Ar}\left(1 s_{5} / 1 s_{3}\right) \rightleftarrows \mathrm{e}^{-}+\operatorname{Ar}\left(1 s_{4} / 1 s_{2}\right)
$$

However, the reverse transfers of reaction 25 have comparable rate coefficients ${ }^{28,29}$ and instead of introducing a net loss of population in metastable states, the electron collision induced transfer reactions should be considered as partial population mixing mechanisms between the four $1 s$ states. With the rate coefficient reported in ${ }^{78}: k_{24}=1.1 \times 10^{-44} \mathrm{~m}^{6} . \mathrm{s}^{-1}$, the 3body quenching frequency of $1 s_{5}$ metastable atoms in pure argon would be $4.5 \times 10^{6} \mathrm{~s}^{-1}$.

Besides volume quenching, the metastable species also diffuse across the gas volume and de-excite when impacting on the reactor walls. The diffusion timescale is given by

$$
\tau_{\text {diff }}=\Lambda^{2} / D_{\text {eff }}
$$

where $\Lambda$, the characteristic length scale is given by $\Lambda=L / \pi$, with $L \approx 300 \mu \mathrm{m}$ being the distance to the electrode of the maximum $1 s_{5}$ metastable density and $D_{\text {eff }}$ the binary diffusion coefficient, whose value is $1.0 \times 10^{-5} \mathrm{~m}^{2} \mathrm{~s}^{-1}$ for $\operatorname{Ar}\left(1 s_{5}\right)$ in atmospheric pressure Ar at $365 \mathrm{~K} .{ }^{78}$ The diffusion time, estimated as $1 \mathrm{~ms}$, is more than 3 orders of magnitude longer than the time for the collisional quenching of $1 s_{5}$ state by 3 body reaction $24(0.2 \mu \mathrm{s})$. Thus, the metastable atoms are primarily lost by collisional processes in the plasma volume. This would also mean that their recorded density profile corresponds exactly to the axial profile of their production rate.

For $1 s_{3}$ metastable atoms, even with slightly different rate coefficients for their production, ${ }^{21}$ quenching, ${ }^{25}$ and state mixing ${ }^{28,29}$ than for $1 s_{5}$ atoms, their kinetics is similar to that of $1 s_{5}$ atoms and except for the absolute values, their density profiles are almost identical. 
Densities of atoms in resonant states ( $1 s_{4}$ and $\left.1 s_{2}\right)$, shown in Fig. 12, are also similar to those of metastable atoms. The production mechanisms for these resonant states include also direct and cascaded electron-impact excitation from the groundstate and $\mathrm{e}-\mathrm{Ar}_{2}^{+}$dissociative recombination. Electron-induced transfers between these states and metastable states $\left(1 s_{5}\right.$ and $1 s_{3}$ ) cannot be considered as a net gain or loss mechanisms for the population of these states. Globally, the $1 s_{5}$ state is receiving more than what is transferred from it to the other $1 s$ states, the fact which, together with its largest statistical weight, explains the highest density of this state. However, the number of atoms transferred in one way and in the opposite way are comparable, within a factor of about 2. As an example, with rate coefficients reported in $^{29}$ and an electron density of $1 \times 10^{19} \mathrm{~m}^{-3}$, the $1 s_{5} \rightarrow 1 s_{4}$ transfer frequency would be $1.9 \times 10^{6} \mathrm{~s}^{-1}$ and $3.1 \times 10^{6} \mathrm{~s}^{-1}$ for the reverse transfer $1 s_{4} \rightarrow 1 s_{5}$. These values are about 2-3 times smaller than the 3-body quenching frequencies of these states. We thus can conclude that, under our experimental conditions, e-impact transfers are not an important process for controlling the density of $1 s$ states.

The radiative lifetimes, $\tau_{\mathrm{rad}}$ of the $1 s_{2}$ and $1 s_{4}$ states are 1.88 and $7.58 \mathrm{~ns},{ }^{51}$ respectively. However, due to the resonance radiation trapping, ${ }^{79,80}$ the effective radiative decay time of population of these states rises to about 0.37 and 1.48 $\mu \mathrm{s}$ for $1 s_{2}$ and $1 s_{4}$ states, respectively, when the pure argon gas medium is approximated by two infinite parallel-plan limited slab of $2 \mathrm{~mm}$ thickness, an acceptable approximation to illustrate radiation trapping for the experimental conditions in our case. These global effective lifetimes, $\tau_{\text {eff }}$ are calculated using the Walsh model, ${ }^{81}$ which is the generalization of the Holstein theory. ${ }^{79,80}$ Atoms in resonance states are also quenched by 3 -body collisions (similar to 24) and rate coefficients reported in ${ }^{78}$ for $1 s_{2}$ and $1 s_{4}$ states are $k_{24}\left(1 s_{2}\right)=$ $2.4 \times 10^{-45} \mathrm{~m}^{6} \mathrm{~s}^{-1}$ and $k_{24}\left(1 s_{4}\right)=1.2 \times 10^{-44} \mathrm{~m}^{6} \mathrm{~s}^{-1}$, respectively. In pure argon at $365 \mathrm{~K}$, they lead to quenching frequencies $v_{\mathrm{q}}$ of about $1.0 \times 10^{6} \mathrm{~s}^{-1}$ and $5.1 \times 10^{6} \mathrm{~s}^{-1}$ for $1 s_{2}$ and $1 s_{4}$ states, respectively. Comparing for each state the global radiative decay frequency, $1 / \tau_{\text {eff }}$ and the 3 -body loss frequency, one can conclude that the photon emission controls the decay of the global density of atoms in the $1 s_{2}$ state but the 3-body collisions are dominant loss mechanism for atoms in the $1 s_{4}$ state.

\section{He $+17 \%$ Ar RF glow discharge}

Fig. 13 shows the spatial distribution of density of atoms in the investigated excited states of argon in 17\% $\mathrm{Ar}$ in $\mathrm{He}$ gas mixture. The density profiles are almost similar to those shown for the pure argon in Fig. 12 and correspond well with the emission profile of the plasma shown in Fig. 1d. Peak density of atoms in metastable $\left(1 s_{3}, 1 s_{5}\right)$ and resonant $\left(1 s_{2}\right.$, $1 s_{4}$ ) states are measured near the sheath region, $400 \mu \mathrm{m}$ away from the electrode surface. The maximum absolute density of slightly larger than $10^{18} \mathrm{~m}^{-3}$ is observed for the $1 s_{5}$ state. Compared to the pure Ar discharge, the peak metastable $1 s_{3}$ and $1 s_{5}$ densities are about two times larger but those of reso-

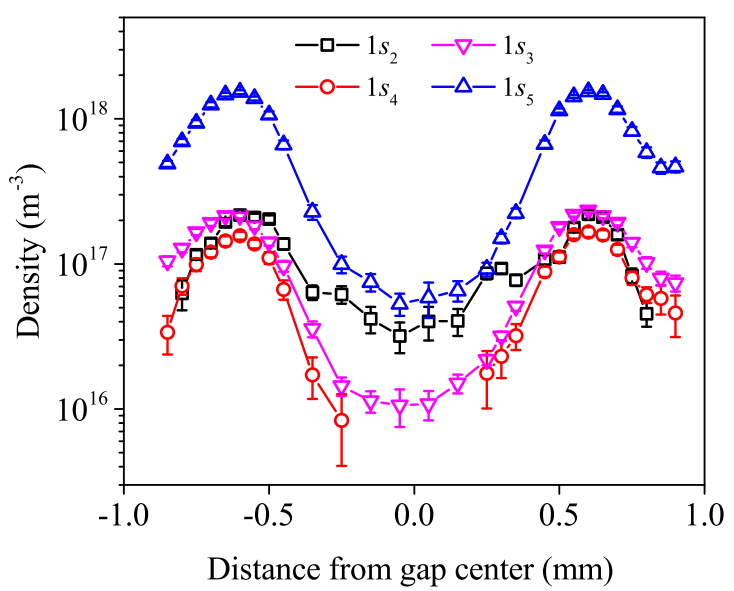

FIG. 13. Time-averaged spatially resolved absolute densities of atoms in $1 s_{2}, 1 s_{3}, 1 s_{4}$ and $1 s_{5}$ states of argon in $\mathrm{He}+17 \%$ Ar plasma at a discharge power of $12.4 \mathrm{~W}$ and total gas flow rate of $1 \mathrm{slm}$.

nance states $1 s_{2}$ and $1 s_{4}$ are almost similar and about one order of magnitude lower than that of the $1 s_{5}$ state. The densities decay when going away from the sheath region and at the central part of the plasma, drop below the detection limit for $1 s_{4}$ resonant state. The peaks seem to be broader than in pure Ar and also slightly farther from electrodes (Fig. 12), which suggests a wider excitation zone. Helium being the main constituent of this plasma, an order of magnitude lowering of the electron density and an enhancement of $T_{\mathrm{e}}$ is expected, relative to the pure argon plasma (see Fig. 10 and Table IV). The main consequences in the kinetics of $\operatorname{Ar}(1 s)$ atoms when switching from argon to $17 \% \mathrm{Ar}+\mathrm{He}$ are twice. First, the e-impact collisional transfer rates between metastable and neighboring resonance states will be much reduced in the mixture, almost decoupling their respective densities. Second, the most frequent 3-body quenching collision will be:

$$
\mathrm{Ar}(1 s)+\mathrm{Ar}+\mathrm{He} \rightarrow \mathrm{Ar}_{2}^{*}+\mathrm{He}
$$

To our knowledge, no experimental or theoretical value for the rate coefficient of this reaction has been reported in the literature. The quenching coefficient of $\operatorname{Ar}\left(1 s_{5}\right)$ metastable atoms by $\mathrm{He}$ must be smaller than by Ar because helium was used as carrier gas in the afterglow experiments of Jones et al for the study of argon metastable atoms reaction with different reactants. ${ }^{82}$ Also, the rate coefficient for the quenching of excited $\mathrm{Ar}^{*}$ atoms by He was found to be smaller, or comparable to their quenching by argon. ${ }^{83}$ In the present case of reactions 24 and 27, the $3^{\text {rd }}$ collision partner (Ar or He) is only acting for the stabilization of the transient $\mathrm{Ar}_{2}^{*}$ excimer formed after $\operatorname{Ar}(1 s)$-Ar encounter. Considering that the $3^{\text {rd }}$ collision partner plays an identical kinetic role in both reactions, we will assume for reaction 27 a similar rate coefficient than in reaction 24 . However, as the gas mixture contains only $17 \%$ of argon, the 3-body quenching frequencies of $\operatorname{Ar}(1 s)$ states will be 0.17 times the above calculated frequencies in pure argon discharge. Taking into account the gas density change 
with the temperature $(365 \mathrm{~K}$ in $\mathrm{Ar}$ and $335 \mathrm{~K}$ in $\mathrm{He}+17 \%$ Ar plasmas), we end up with 3-body collision quenching frequencies $v_{\mathrm{q}}$ of $2.0 \times 10^{5} \mathrm{~s}^{-1}$ and $1.0 \times 10^{6} \mathrm{~s}^{-1}$ for $1 s_{2}$ and $1 s_{4}$ states, respectively. However, in $\mathrm{He}+17 \%$ Ar mixture, the effective radiative lifetimes predicted by the radiation trapping model ${ }^{79,80}$ remain unchanged, compared to the pure argon case. In fact, at atmospheric pressure the partial density of argon in the mixture is high enough for the spectral profile of the resonance lines 104.7 and $106.7 \mathrm{~nm}$ being still controlled by the resonance broadenings of their upper $1 s_{2}$ and $1 s_{4}$ states, respectively, and for the effective radiative lifetimes, predicted by the radiation trapping model, having reached their asymptotic highest values. ${ }^{79,80}$

In $\mathrm{He}+17 \%$ Ar discharge, the most remarkable difference between the density distribution profiles of atoms in the four $1 s$ states, but also with the pure Ar case, is the much slower decay of the density of $\operatorname{Ar}\left(1 s_{2}\right)$ atoms toward the plasma center. In the middle of electrodes, the density of $\operatorname{Ar}\left(1 s_{2}\right)$ atoms is only 5 times smaller than its peak value at the sheath region but the relative change for metastable $\operatorname{Ar}\left(1 s_{5}\right)$ density is about 30 (Fig. 13). This peculiar behavior of $\operatorname{Ar}\left(1 s_{2}\right)$ atoms density in the $\mathrm{He}+17 \%$ Ar gas mixture is attributed to the smallness of the 3-body quenching rate of the $1 s_{2}$ state, which permits the spatial transport of the excitation in this state by radiation trapping phenomenon. To verify this assertion, a collisional radiative model, based on the random walk of resonance photon by Monte Carlo statistic was developed as discussed in Section II H and the results are discussed below.

\section{Excitation transport by resonance radiation}

Fig. 14 represents the measured and simulated densities of atoms in $1 s_{2}$ and $1 s_{4}$ states in the pure argon discharge. The simulation is performed as described in detail in Section II H. The peak amplitudes are normalized to 1 and, in the simulation, the plasma excitation zone of $0.14 \mathrm{~mm}$ wide was set between 0.2 and $0.34 \mathrm{~mm}$ from electrodes (positions \pm 0.66 to $\pm 0.8 \mathrm{~mm})$.

At the gap center, the simulated density of $1 s_{2}$ atoms is larger than that of $1 s_{4}$ atoms because the former being less exposed to the 3-body quenching collisions during their short radiative lifetime, the $104.8 \mathrm{~nm}$ photons can propagate farther from their production zone and create $1 s_{2}$ atoms in the central part of the gap. However, the uncertainty level on measured densities in positions far from the sheaths does not permit an indisputable conclusion for the agreement between experimental and simulated density profiles. Nonetheless, the different behavior of $1 s_{2}$ and $1 s_{4}$ atoms as found in the experiment in the $\mathrm{He}+17 \%$ Ar mixture is clearly reproduced by the model (Fig. 15). In the simulated profiles, the plasma excitation zone of $0.20 \mathrm{~mm}$ wide was set between 0.3 and $0.5 \mathrm{~mm}$ from electrodes (positions \pm 0.5 to $\pm 0.7 \mathrm{~mm}$ ). Thanks to the larger density of excited atoms in this discharge, the signal-tonoise ratio of the experimental data points is much better and a good agreement with simulated profiles is observed. It should be pointed out that with about 5 times smaller 3-body quenching rates in this plasma, compared to pure argon discharge,

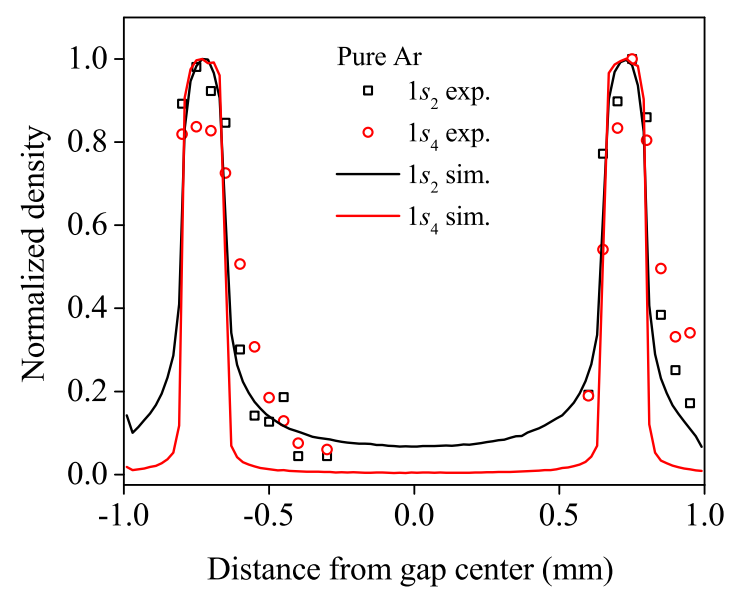

FIG. 14. Measured and simulated normalized densities of atoms in $1 s_{2}$ and $1 s_{4}$ resonant states in the pure Ar plasma.

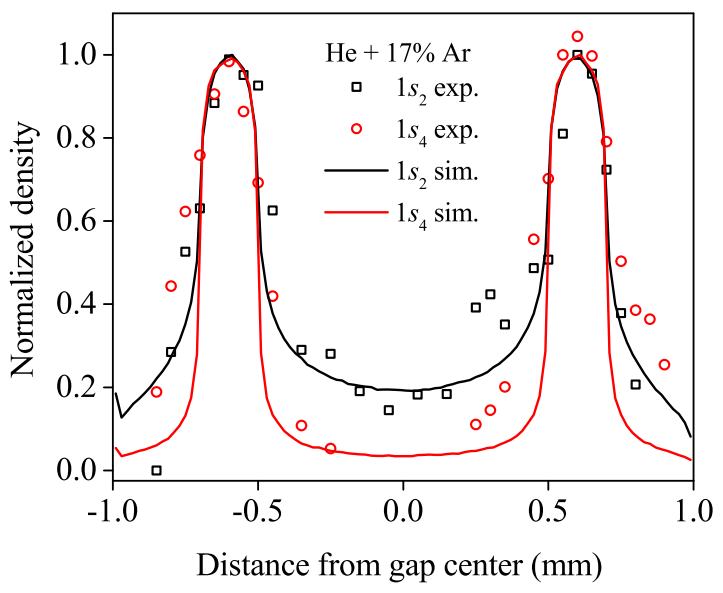

FIG. 15. Measured and simulated normalized densities of atoms in $1 s_{2}$ and $1 s_{4}$ resonant states in the $\mathrm{He}+17 \%$ Ar plasma.

the resonance photons can diffuse farther from the sheath regions and transport the excitation in the resonance states to the central part of the plasma volume. We also note that the ratio between the quenching frequency $\left(v_{\mathrm{q}}\right)$ and the radiative decay frequency $\left(1 / \tau_{\text {rad }}\right)$ being 20 times smaller in the mixture for $1 s_{2}$ atoms than for $1 s_{4}$ ones $\left(3.8 \times 10^{-4}\right.$ and $7.6 \times 10^{-3}$, respectively), the excitation transport by $104.8 \mathrm{~nm}$ photons is more efficient than by $106.7 \mathrm{~nm}$ photons.

\section{E. Comparison with He RF glow discharge}

The RF glow discharges operated in He have been intensively studied both experimentally and theoretically, ${ }^{67,76,84}$ while a diffuse RF glow discharge in Ar has not been investigated in detail to date. Unlike glow discharges in He, the gas temperature is higher and the emission intensity is stronger in pure Ar discharge. The dominant mode of ionization in $\mathrm{He}$ 
discharge is through Penning ionization of impurities present in the gas such as $\mathrm{N}_{2}, \mathrm{O}_{2}$ or $\mathrm{H}_{2} \mathrm{O}$. However, the energy in the metastable state of $\mathrm{Ar}$ is too low to enable Penning ionization. Considering a Maxwellian EEDF, most ionization would occur from direct electron impact of ground-state Ar atoms with an ionization rate of $1.6 \times 10^{25} \mathrm{~m}^{-3} \mathrm{~s}^{-1}, 38$ however, if the EEDF is non-Maxwellian, the dominant ionization mechanism for the estimated electron temperature of $2.1 \mathrm{eV}$ from the continuum emission measurement needs to be stepwise ionization with an ionization rate of $1.0 \times 10^{23} \mathrm{~m}^{-3} \mathrm{~s}^{-138}$

$$
\mathrm{e}^{-}+\operatorname{Ar}\left(1 s_{5}\right) \rightarrow \mathrm{Ar}^{+}+2 \mathrm{e}^{-}
$$

Associative ionization,

$$
\operatorname{Ar}\left(1 s_{5}\right)+\operatorname{Ar}\left(1 s_{5}\right) \rightarrow \operatorname{Ar}+\mathrm{Ar}+\mathrm{e}^{-}
$$

with a rate coefficient of $6.4 \times 10^{-16} \mathrm{~m}^{3} \mathrm{~s}^{-1}, 77$ has an ionization rate that is 2 orders of magnitude smaller than the stepwise ionization 28. A brief comparison of the measured plasma properties of Ar discharge reported in this paper with He RF glow discharge is summarized in Table VI.

\section{CONCLUSION}

In this work, a radio frequency-driven capacitively coupled argon glow discharge at atmospheric pressure is investigated. We deduce the electron temperature and density by fitting the absolute intensity of the recorded emission spectrum with the stimulated spectrum of the neutral bremsstrahlung radiation. Using a non-Maxwellian EEDF, the time and space-averaged $T_{\mathrm{e}}$ and $n_{\mathrm{e}}$ of of $2.1 \mathrm{eV}$ and $1.1 \times 10^{19} \mathrm{~m}^{-3}$ were estimated, respectively, for pure Ar plasma. Using similar approach for a diffuse He RF discharge, a $T_{\mathrm{e}}$ of $3.5 \mathrm{eV}$ and an $n_{\mathrm{e}}$ of $1.2 \times 10^{17}$ $\mathrm{m}^{-3}$ were obtained.

We also have applied for the first time broadband absorption spectroscopy for the measurement of the space-resolved absolute density of atoms in metastable $\left(1 s_{5}\right.$ and $\left.1 s_{3}\right)$ and resonant $\left(1 s_{4}\right.$ and $\left.1 s_{2}\right)$ states of argon in a $2 \mathrm{~mm}$ wide plasma reactor running in pure argon or $\mathrm{Ar} / \mathrm{He}$ mixture at atmospheric pressure. In all the discharge conditions, the density profiles of these excited atoms across the plasma gap showed features similar to the light emission profile of a glow RF discharge operated in the $\alpha$-mode at atmospheric pressure. A maximum density of the order of $10^{18} \mathrm{~m}^{-3}$ was obtained for the $1 s_{5}$ metastable atoms, while in the other metastable state $\left(1 s_{3}\right)$ and resonant states, $1 s_{4}$ and $1 s_{2}$, maximum densities were $\sim$ $2 \times 10^{17} \mathrm{~m}^{-3}$, which occurred in the sheath region. The major production mechanisms of metastable atoms are the electronimpact excitation from the ground state and the dissociative electron-ion recombination of the $\mathrm{Ar}_{2}^{+}$ions. Their dominant loss mechanism is through the three-body collisional quenching with 2 ground state argon atoms, forming $\mathrm{Ar}_{2}^{*} \mathrm{ex}-$ cimer. Beside the above cited production and loss mechanisms, atoms in the resonance states are also lost by radiative decay to the ground state, controlled by the resonance radiation trapping process in the optically thick medium. While the density of atoms in three $1 s_{5}, 1 s_{4}$ and $1 s_{3}$ states drops by factor 10 within $200 \mu \mathrm{m}$ aside their production zone in the sheath region and becomes hardly detectable, the density of $1 s_{2}$ resonance atoms in the center of the $\mathrm{He}+17 \%$ Ar discharge is about $20 \%$ of its peak value in the sheath. This peculiar behavior is attributed to the smaller 3-body quenching rate coefficient of $1 s_{2}$ atoms, compared to the 3 other $1 s$ atoms and its shorter radiative lifetime, relative to that of the $1 s_{4}$ state. This assertion is confirmed by comparing the measured density profiles of atoms in the resonance states to the density profiles calculated with a kinetic Monte Carlo statistic code of the random walk of the resonance photons in optically thick medium. A detailed comparison of the key plasma properties measured for both He and Ar RF glow discharges shows, for the same power density, significantly larger $T_{\mathrm{e}}$ for He compared to Ar, while the electron density is more than an order of magnitude higher in Ar than in helium.

\section{ACKNOWLEDGMENTS}

This work was supported by the US Department of Energy, Office of Science, Office of Fusion Energy Sciences General Plasma Science program under Award Number AT4010100 and DE-SC-0020232 and by the NSF grant under Award Number PHYS 1903151. GN gratefully acknowledges the Kermit and Ione Ebeltoft Interdisciplinary Doctoral Fellowship for 2018-19 and the Doctoral Dissertation Fellowship for 2019-20.

\section{DATA AVAILABILITY}

The data that support the findings of this study are available from the corresponding author upon reasonable request.

${ }^{1}$ J. Han and M. C. Heaven, "Kinetics of optically pumped Ar metastables," Optics Letters 39, 6541-6544 (2014).

${ }^{2}$ D. Emmons and D. Weeks, "Kinetics of high pressure argon-helium pulsed gas discharge," Journal of Applied Physics 121, 203301 (2017).

${ }^{3}$ D. Emmons, D. Weeks, B. Eshel, and G. Perram, "Metastable $\operatorname{Ar}\left(1 s_{5}\right)$ density dependence on pressure and argon-helium mixture in a high pressure radio frequency dielectric barrier discharge," Journal of Applied Physics 123, 043304 (2018).

${ }^{4}$ A. R. Hoskinson, J. Gregorío, J. Hopwood, K. Galbally-Kinney, S. J. Davis, and W. T. Rawlins, "Argon metastable production in argon-helium microplasmas," Journal of Applied Physics 119, 233301 (2016).

${ }^{5}$ A. R. Hoskinson, J. Gregorío, J. Hopwood, K. L. Galbally-Kinney, S. J. Davis, and W. T. Rawlins, "Spatially resolved modeling and measurements of metastable argon atoms in argon-helium microplasmas," Journal of Applied Physics 121, 153302 (2017)

${ }^{6}$ G. Isbary, G. Morfill, H. Schmidt, M. Georgi, K. Ramrath, J. Heinlin, S. Karrer, M. Landthaler, T. Shimizu, B. Steffes, et al., "A first prospective randomized controlled trial to decrease bacterial load using cold atmospheric argon plasma on chronic wounds in patients," British Journal of Dermatology 163, 78-82 (2010).

${ }^{7}$ C. Van Gils, S. Hofmann, B. Boekema, R. Brandenburg, and P. Bruggeman, "Mechanisms of bacterial inactivation in the liquid phase induced by a remote RF cold atmospheric pressure plasma jet," Journal of Physics D: Applied Physics 46, 175203 (2013). 
TABLE VI. Comparison of RF glow discharges in He and Ar.

\begin{tabular}{lll}
\hline \hline Parameter & He & Ar \\
\hline Ionization & Penning ionization & Stepwise (metastable) ionization \\
Metastable atoms/molecules $^{\mathrm{a}}$ & $2^{3} S\left((6.2 \pm 0.4) \times 10^{18} \mathrm{~m}^{-3}\right)$, & $1 s_{5}\left((6.9 \pm 0.4) \times 10^{17} \mathrm{~m}^{-3}\right)$, \\
& $a^{3} \Sigma_{\mathrm{u}}^{+}\left((2.5 \pm 0.6) \times 10^{18} \mathrm{~m}^{-3}\right)^{31}$ & $1 s_{3}\left((1.13 \pm 0.12) \times 10^{17} \mathrm{~m}^{-3}\right)$ \\
Resonant atoms $^{\mathrm{a}}$ & - & $1 s_{4}\left((1.72 \pm 0.08) \times 10^{17} \mathrm{~m}^{-3}\right)$, \\
& & $1 s_{2}\left((2.12 \pm 0.11) \times 10^{17} \mathrm{~m}^{-3}\right)$ \\
Power density & $38.6 \mathrm{Wcm}^{-3}$ & $37.2 \mathrm{Wcm}^{-3}$ \\
Sheath width & $160 \pm 10 \mu \mathrm{m}^{\mathrm{b}}$ & $170 \pm 10 \mu \mathrm{m}^{\mathrm{b}}$ \\
& $380 \pm 41 \mu \mathrm{m}^{\mathrm{c}}$ & $180 \pm 9 \mu \mathrm{m}^{\mathrm{c}}$ \\
RF mode & $\alpha-\mathrm{mode}$ & $\alpha-\mathrm{mode}$ \\
$n_{\mathrm{e}}$ & $(1.2 \pm 0.2) \times 10^{17} \mathrm{~m}^{-3}$ & $(1.1 \pm 0.7) \times 10^{19} \mathrm{~m}^{-3}$ \\
$T_{\mathrm{e}}$ & $(3.5 \pm 0.4) \mathrm{eV}$ & $(2.1 \pm 0.3) \mathrm{eV}$ \\
\hline \hline
\end{tabular}

a Maximum densities measured in the sheath region of the discharge.

${ }^{\mathrm{b}}$ From optical imaging using digital camera.

${ }^{\mathrm{c}}$ From equivalent electrical model.

${ }^{8}$ K. Wende, P. Williams, J. Dalluge, W. Van Gaens, H. Aboubakr, J. Bischof, T. Von Woedtke, S. M. Goyal, K.-D. Weltmann, A. Bogaerts, et al., "Identification of the biologically active liquid chemistry induced by a nonthermal atmospheric pressure plasma jet," Biointerphases 10, 029518 (2015).

${ }^{9}$ S. Canulescu, J. Whitby, K. Fuhrer, M. Hohl, M. Gonin, T. Horvath, and J. Michler, "Potential analytical applications of negative ions from a pulsed radiofrequency glow discharge in argon," Journal of Analytical Atomic Spectrometry 24, 178-180 (2009).

${ }^{10}$ S. K. Øiseth, A. Krozer, B. Kasemo, and J. Lausmaa, "Surface modification of spin-coated high-density polyethylene films by argon and oxygen glow discharge plasma treatments," Applied Surface Science 202, 92-103 (2002).

${ }^{11}$ N. Gomathi and S. Neogi, "Surface modification of polypropylene using argon plasma: Statistical optimization of the process variables," Applied Surface Science 255, 7590-7600 (2009).

${ }^{12} \mathrm{Z}$. Machala, K. Hensel, and Y. Akishev, Plasma for bio-decontamination, medicine and food security (Springer Science \& Business Media, 2012).

${ }^{13}$ K. Stapelmann, O. Kylián, B. Denis, and F. Rossi, "On the application of inductively coupled plasma discharges sustained in $\mathrm{Ar} / \mathrm{O}_{2} / \mathrm{N}_{2}$ ternary mixture for sterilization and decontamination of medical instruments," Journal of Physics D: Applied Physics 41, 192005 (2008).

${ }^{14}$ C. Hertwig, K. Reineke, J. Ehlbeck, D. Knorr, and O. Schlüter, "Decontamination of whole black pepper using different cold atmospheric pressure plasma applications," Food Control 55, 221-229 (2015).

${ }^{15}$ S. De Benedictis and G. Dilecce, "Energy transfers by long-lived species in glows and afterglows," Pure and Applied Chemistry 74, 317-326 (2002).

${ }^{16}$ A. Bogaerts, R. Gijbels, and J. Vlcek, "Collisional-radiative model for an argon glow discharge,” Journal of Applied Physics 84, 121-136 (1998).

${ }^{17} \mathrm{~S}$. Rauf and M. J. Kushner, "Argon metastable densities in radio frequency $\mathrm{Ar}, \mathrm{Ar} / \mathrm{O}_{2}$ and $\mathrm{Ar} / \mathrm{CF}_{4}$ electrical discharges," Journal of Applied Physics 82, 2805-2813 (1997).

${ }^{18}$ X.-M. Zhu and Y.-K. Pu, "A simple collisional-radiative model for lowtemperature argon discharges with pressure ranging from $1 \mathrm{~Pa}$ to atmospheric pressure: kinetics of Paschen 1s and 2p levels,' Journal of Physics D: Applied Physics 43, 015204 (2009).

${ }^{19}$ A. Bogaerts and R. Gijbels, "Description of the argon-excited levels in a radio-frequency and direct current glow discharge," Spectrochimica Acta Part B: Atomic Spectroscopy 55, 263-278 (2000).

${ }^{20}$ W. Van Gaens and A. Bogaerts, "Kinetic modelling for an atmospheric pressure argon plasma jet in humid air," Journal of Physics D: Applied Physics 46, 275201 (2013)

${ }^{21} \mathrm{~K}$. Tachibana, "Excitation of the $1 s_{5}, 1 s_{4}, 1 s_{3}$, and $1 s_{2}$ levels of argon by low-energy electrons," Physical Review A 34, 1007 (1986).

${ }^{22}$ B. Niermann, M. Böke, N. Sadeghi, and J. Winter, "Space resolved density measurements of argon and helium metastable atoms in radio-frequency generated He-Ar micro-plasmas," The European Physical Journal D 60, 489-495 (2010)
${ }^{23}$ B. Eshel, C. A. Rice, and G. P. Perram, "Pressure broadening and shift rates for $\operatorname{Ar}(\mathrm{s}-\mathrm{p})$ transitions observed in an Ar-He discharge," Journal of Quantitative Spectroscopy and Radiative Transfer 179, 40-50 (2016).

${ }^{24}$ I. Stefanović, T. Kuschel, S. Schröter, and M. Böke, "Argon metastable dynamics and lifetimes in a direct current microdischarge," Journal of Applied Physics 116, 113302 (2014).

${ }^{25}$ S. G. Belostotskiy, V. M. Donnelly, D. J. Economou, and N. Sadeghi, "Spatially resolved measurements of argon metastable $\left(1 s_{5}\right)$ density in a high pressure microdischarge using diode laser absorption spectroscopy," IEEE Transactions on Plasma Science 37, 852-858 (2009).

${ }^{26}$ M. Schulze, A. Yanguas-Gil, A. Von Keudell, and P. Awakowicz, "A robust method to measure metastable and resonant state densities from emission spectra in argon and argon-diluted low pressure plasmas," Journal of Physics D: Applied Physics 41, 065206 (2008).

${ }^{27} \mathrm{G}$. Hebner, "Spatially resolved, excited state densities and neutral and ion temperatures in inductively coupled argon plasmas," Journal of Applied Physics 80, 2624-2636 (1996).

${ }^{28}$ E. Carbone, E. Van Veldhuizen, G. Kroesen, and N. Sadeghi, "Electron impact transfer rates between metastable and resonant states of argon investigated by laser pump-probe technique," Journal of Physics D: Applied Physics 48, 425201 (2015).

${ }^{29}$ E. Carbone, S. Hübner, J. Van Der Mullen, G. Kroesen, and N. Sadeghi, "Determination of electron-impact transfer rate coefficients between argon $1 s_{2}$ and $1 s_{3}$ states by laser pump-probe technique," Journal of Physics D: Applied Physics 46, 415202 (2013).

${ }^{30}$ J. Golda, F. Kogelheide, P. Awakowicz, and V. Schulz-von der Gathen, "Dissipated electrical power and electron density in an RF atmospheric pressure helium plasma jet," Plasma Sources Science and Technology 28, 095023 (2019).

${ }^{31}$ G. Nayak, N. Sadeghi, and P. Bruggeman, "He$\left(2^{3} S_{1}\right)$ and $\operatorname{He}_{2}\left(a^{3} \Sigma_{\mathrm{u}}^{+}\right)$ metastables densities measured in an RF-driven helium plasma using broadband absorption spectroscopy," Plasma Sources Science and Technology 28, 125006 (2019).

${ }^{32}$ G. Oinuma, G. Nayak, Y. Du, and P. J. Bruggeman, "Controlled plasmadroplet interactions: a quantitative study of $\mathrm{OH}$ transfer in plasma-liquid interaction," Plasma Sources Science and Technology 29, 095002 (2020).

${ }^{33}$ S. Park, W. Choe, H. Kim, and J. Y. Park, "Continuum emission-based electron diagnostics for atmospheric pressure plasmas and characteristics of nanosecond-pulsed argon plasma jets," Plasma Sources Science and Technology 24, 034003 (2015).

${ }^{34}$ S. Park, W. Choe, S. Youn Moon, and J. Park, "Electron density and temperature measurement by continuum radiation emitted from weakly ionized atmospheric pressure plasmas," Applied Physics Letters 104, 084103 (2014).

${ }^{35}$ D. Hammer and L. Frommhold, "Polarization bremsstrahlung spectra of electron-rare-gas atom collisions at temperatures from 5 to $40 \mathrm{kK}$," Physical Review A 64, 024705 (2001). 
${ }^{36}$ A. Z. Akcasu and L. H. Wald, "Bremsstrahlung of slow electrons in neutral gases and free-free absorption of microwaves," The Physics of Fluids 10, 1327-1335 (1967).

${ }^{37}$ K. T. A. L. Burm, "Continuum radiation in a high pressure argon-mercury lamp," Plasma Sources Science and Technology 13, 387 (2004).

${ }^{38}$ G. Hagelaar and L. Pitchford, "Solving the Boltzmann equation to obtain electron transport coefficients and rate coefficients for fluid models," Plasma Sources Science and Technology 14, 722 (2005).

${ }^{39}$ V. Kas'yanov and A. Starostin, "On the theory of bremsstrahlung of slow electrons on atoms," Soviet Physics JETP 21, 15 (1965).

40 "Phelps database," http: //www. Ixcat. net, retrieved on June 17, 2020.

${ }^{41}$ L. Taghizadeh, A. Nikiforov, R. Morent, J. van der Mullen, and C. Leys, "Determination of the electron temperature of atmospheric pressure argon plasmas by absolute line intensities and a collisional radiative model," Plasma Processes and Polymers 11, 777-786 (2014).

${ }^{42}$ Q. Xiong, A. Y. Nikiforov, M. A. González, C. Leys, and X. P. Lu, "Characterization of an atmospheric helium plasma jet by relative and absolute optical emission spectroscopy," Plasma Sources Science and Technology 22, 015011 (2012).

${ }^{43}$ K. McKay, F. Iza, and M. Kong, "Excitation frequency effects on atmospheric-pressure helium RF microplasmas: plasma density, electron energy and plasma impedance," The European Physical Journal D 60, 497503 (2010).

${ }^{44}$ M. A. Lieberman and A. J. Lichtenberg, Principles of plasma discharges and materials processing, 2nd ed. (John Wiley \& Sons, 2005).

${ }^{45}$ P. Chabert and N. Braithwaite, Physics of radio-frequency plasmas (Cambridge University Press, 2011).

${ }^{46}$ Y. P. Raizer, Gas Discharge Physics (Springer, 1991).

${ }^{47}$ Y. Du, G. Nayak, G. Oinuma, Y. Ding, Z. Peng, and P. J. Bruggeman, "Emission considering self-absorption of $\mathrm{OH}$ to simultaneously obtain the $\mathrm{OH}$ density and gas temperature: validation, non-equilibrium effects and limitations," Plasma Sources Science and Technology 26, 095007 (2017).

${ }^{48}$ P. Bruggeman, G. Cunge, and N. Sadeghi, "Absolute OH density measurements by broadband UV absorption in diffuse atmospheric-pressure $\mathrm{He}-\mathrm{H}_{2} \mathrm{O}$ rf glow discharges," Plasma Sources Science and Technology 21, 035019 (2012).

${ }^{49}$ M. Kogelschatz, G. Cunge, and N. Sadeghi, "Identification of halogen containing radicals in silicon etching plasmas and density measurement by UV broad band absorption spectroscopy," Journal of Physics D: Applied Physics 37, 1954 (2004)

${ }^{50}$ Y. Du, G. Nayak, G. Oinuma, Z. Peng, and P. J. Bruggeman, "Effect of water vapor on plasma morphology, $\mathrm{OH}$ and $\mathrm{H}_{2} \mathrm{O}_{2}$ production in $\mathrm{He}$ and Ar atmospheric pressure dielectric barrier discharges," Journal of Physics D: Applied Physics 50, 145201 (2017).

${ }^{51}$ A. Kramida, Y. Ralchenko, J. Reader, and NIST ASD Team, NIST Atomic Spectra Database (ver. 5.7.1), [Online]. Available: https://physics . nist.gov/asd [2019, October 20]. National Institute of Standards and Technology, Gaithersburg, MD. (2019).

${ }^{52}$ H. L. Anderson, A physicist's desk reference, 2nd ed. (New York: American Institute of Physics (AIP), 1989).

${ }^{53}$ C. Lee, D. Camm, and G. Copley, "Van der Waals broadening of argon absorption lines," Journal of Quantitative Spectroscopy and Radiative Transfer 15, 211-216 (1975).

${ }^{54}$ J. Rosato, Y. Marandet, D. Reiter, and R. Stamm, "Development of a hybrid kinetic-fluid model for line radiation transport in magnetic fusion plasmas," High Energy Density Physics 22, 73-76 (2017).

${ }^{55} \mathrm{~J}$. Rosato, "Derivation of a transport equation for line radiation using the Wigner Phase Space Formalism,' Journal of Computational and Theoretical Transport 47, 18-27 (2018).

${ }^{56} \mathrm{~J}$. Spanier and E. M. Gelbard, Monte Carlo principles and neutron transport problems (Courier Corporation, 2008).

${ }^{57}$ C. Yamabe, S. Buckman, and A. Phelps, "Measurement of free-free emission from low-energy-electron collisions with Ar," Physical Review A 27, 1345 (1983).

${ }^{58}$ I. Denysenko, H. Kersten, and N. Azarenkov, "Electron energy distribution in a dusty plasma: analytical approach,” Physical Review E 92, 033102 (2015).

${ }^{59}$ V. I. Kolobov and V. A. Godyak, "Nonlocal electron kinetics in collisional gas discharge plasmas," IEEE Transactions on Plasma Science 23, 503-531 (1995).
${ }^{60}$ J. Golda, J. Held, and V. Schulz-von der Gathen, "Comparison of electron heating and energy loss mechanisms in an RF plasma jet operated in argon and helium," Plasma Sources Science and Technology 29, 025014 (2020).

${ }^{61}$ J. Gudmundsson, “Technical Report RH-21-2002 Science Institute," University of Iceland (2002).

${ }^{62}$ D. Levko and L. L. Raja, "Electron kinetics in atmospheric-pressure argon and nitrogen microwave microdischarges," Journal of Applied Physics 119 163303 (2016).

${ }^{63}$ M. Atanasova, A. Sobota, W. Brok, G. Degrez, and J. Van Der Mullen, "Driving frequency dependence of capacitively coupled plasmas in atmospheric argon,” Journal of Physics D: Applied Physics 45, 335201 (2012).

${ }^{64}$ M. Atanasova, E. Carbone, D. Mihailova, E. Benova, G. Degrez, and J. Van Der Mullen, "Modelling of an RF plasma shower," Journal of Physics D: Applied Physics 45, 145202 (2012).

${ }^{65}$ M. Moravej, X. Yang, G. Nowling, J. Chang, R. Hicks, and S. Babayan, "Physics of high-pressure helium and argon radio-frequency plasmas," Journal of Applied Physics 96, 7011-7017 (2004).

${ }^{66}$ N. Balcon, G. Hagelaar, and J. Boeuf, "Numerical model of an argon atmospheric pressure RF discharge," IEEE Transactions on Plasma Science 36, 2782-2787 (2008).

${ }^{67}$ J. Shi and M. Kong, "Mechanisms of the $\alpha$ and $\gamma$ modes in radio-frequency atmospheric glow discharges," Journal of Applied Physics 97, 023306 (2005).

${ }^{68}$ Y. B. Golubovskii, V. Maiorov, J. Behnke, and J. Behnke, "Modelling of the homogeneous barrier discharge in helium at atmospheric pressure," Journal of Physics D: Applied Physics 36, 39 (2002).

${ }^{69}$ Y. Wang and D. Wang, "Influence of impurities on the uniform atmosphericpressure discharge in helium," Physics of Plasmas 12, 023503 (2005).

${ }^{70} \mathrm{G}$. Copley and D. Camm, "Pressure broadening and shift of argon emission lines," Journal of Quantitative Spectroscopy and Radiative Transfer 14, 899-907 (1974).

${ }^{71}$ D. Aeschliman, R. Hill, and D. Evans, "Collisional broadening and shift of neutral argon spectral lines," Physical Review A 14, 1421 (1976).

${ }^{72} \mathrm{D}$. Stacey and J. Vaughan, "Pressure broadening and oscillator strengths in argon,” Physics Letters 11, 105-106 (1964).

${ }^{73} \mathrm{P}$. Moussounda and P. Ranson, "Pressure broadening of argon lines emitted by a high-pressure microwave discharge (Surfatron)," Journal of Physics B: Atomic and Molecular Physics 20, 949 (1987).

${ }^{74} \mathrm{O}$. Vallee, P. Ranson, and J. Chapelle, "Measurements of broadening of argon lines and oscillator strengths of resonance lines," Journal of Quantitative Spectroscopy and Radiative Transfer 18, 327-336 (1977).

${ }^{75}$ N. Konjević, A. Lesage, J. R. Fuhr, and W. L. Wiese, "Experimental Stark widths and shifts for spectral lines of neutral and ionized atoms (a critical review of selected data for the period 1989 through 2000),' Journal of Physical and Chemical Reference Data 31, 819-927 (2002).

${ }^{76}$ T. Gans, C. C. Lin, V. Schulz-Von Der Gathen, and H. Döbele, "Phaseresolved emission spectroscopy of a hydrogen rf discharge for the determination of quenching coefficients," Physical Review A 67, 012707 (2003).

${ }^{77}$ J. T. Gudmundsson and E. Thorsteinsson, "Oxygen discharges diluted with argon: dissociation processes," Plasma Sources Science and Technology 16, 399 (2007).

${ }^{78} \mathrm{~J}$. Kolts and D. Setser, "Decay rates of $\operatorname{Ar}\left(4 s,{ }^{3} P_{2}\right), \operatorname{Ar}\left(4 s^{\prime},{ }^{3} P_{0}\right), \operatorname{Kr}\left(5 s,{ }^{3} P_{2}\right)$, and $\mathrm{Xe}\left(6 s,{ }^{3} P_{2}\right)$ atoms in argon," The Journal of Chemical Physics 68, 4848-4859 (1978).

${ }^{79} \mathrm{~T}$. Holstein, "Imprisonment of resonance radiation in gases," Physical Review 72, 1212 (1947).

${ }^{80} \mathrm{~T}$. Holstein, "Imprisonment of resonance radiation in gases. II," Physical Review 83, 1159 (1951).

${ }^{81} \mathrm{P}$. Walsh, "Effect of simultaneous Doppler and collision broadening and of hyperfine structure on the imprisonment of resonance radiation," Physical Review 116, 511 (1959).

${ }^{82}$ M. T. Jones, T. Dreiling, D. Setser, and R. N. McDonald, "Branching fractions for penning ionization in quenching of $\operatorname{He}\left(2^{3} \mathrm{~S}\right), \operatorname{Ar}\left({ }^{3} \mathrm{P}_{2,0}\right)$, and $\mathrm{Ne}\left({ }^{3} \mathrm{P}_{2,0}\right)$ atoms," The Journal of Physical Chemistry 89, 4501-4517 (1985).

${ }^{83}$ N. Sadeghi, D. Setser, A. Francis, U. Czarnetzki, and H. Döbele, "Quenching rate constants for reactions of $\operatorname{Ar}\left(4 \mathrm{p}\right.$ ' $[1 / 2]_{0}, 4 \mathrm{p}[1 / 2]_{0}, 4 \mathrm{p}[3 / 2]_{2}$, and $4 \mathrm{p}[5 / 2]_{2}$ ) atoms with 22 reagent gases," The Journal of Chemical Physics 115, 3144-3154 (2001). 
${ }^{84}$ J. Park, I. Henins, H. Herrmann, G. Selwyn, and R. Hicks, "Discharge phenomena of an atmospheric pressure radio-frequency capacitive plasma source,” Journal of Applied Physics 89, 20-28 (2001). 